seiner Entscheidung von vornherein vereiteln würde, ist auch die Beschlussfassung durch ein kleineres Gremium denkbar.

Die Finanzkrise, die Europa erfasst hat, mag exzeptionell sein. Die vielzitierten „Märkte“ kommen in den Institutionen des Verfassungsstaates Deutschland nicht vor. Das Bundesverfassungsgericht widersetzt sich indes einer angeblich erforderlichen Reduktion und Komprimierung parlamentarischer Rechte zugunsten einer Organisation des Krisenmanagements, die tragende Prinzipien der repräsentativen Demokratie schwächt. Der Bundestag hat inzwischen den Vorgaben des Bundesverfassungsgerichts entsprochen und seine fehlerhafte Gesetzgebung korrigiert.

\title{
Die Mitwirkung des Bundestages in EU-Angelegenheiten nach dem EUZBBG in der Praxis - ein Kurzkommentar
}

\author{
Hinrich Schröder*
}

Das Gesetz über die Zusammenarbeit von Deutschem Bundestag und Bundesregierung in Angelegenheiten der Europäischen Union ${ }^{1}$ - kurz EUZBBG - konkretisiert die in Art. 23 Abs. 2 und 3 GG verankerten Rechte des Bundestages gegenüber der Bundesregierung auf Unterrichtung und zur Abgabe von Stellungnahmen. Es ermöglicht dem Bundestag, das Handeln der Bundesregierung auf EU-Ebene, insbesondere im Rat, zu kontrollieren. Daneben versetzt das EUZBBG das deutsche Parlament aber auch in die Lage, seinen in Art. 12 EUV $^{2}$ vorgesehenen Beitrag zur guten Arbeitsweise der Europäischen Union zu leisten. ${ }^{3}$

Das EUZBBG wurde anlässlich des Urteils des Bundesverfassungsgerichts zum Vertrag von Lissabon 2009 vollständig überarbeitet. ${ }^{4}$ Ausgangspunkt des Bundesverfassungsgerichts für seine Forderung nach verstärkter Mitwirkung des Bundestages in EU-Angelegenheiten war die neu geschaffene Figur der Integrationsverantwortung. ${ }^{5}$ Diese kommt insbesondere in Fällen zum Tragen, in denen das Primärrecht der EU „allein oder maßgeblich durch die Organe der Union" geändert werden kann. ${ }^{6}$ Dem Gesetzgeber wurden detaillierte Vorgaben gemacht, wie in bestimmten Fallgruppen durch Gesetz beziehungsweise Beschluss mitzuwirken ist. ${ }^{7}$ Diese Vorgaben sind mit dem Integrationsverantwortungsgesetz ${ }^{8}$ umgesetzt worden. Integrationsverantwortung nimmt der Bundestag aber auch wahr, wenn er von

* Der Autor ist Referent im Referat Europa der Verwaltung des Deutschen Bundestages. Der Beitrag gibt ausschließlich seine persönliche Auffassung wieder.

1 Vgl. BGBl. 2009 I, S. 3026.

2 Vgl. ABl. EU Nr. C 83 vom 30. März 2010, S. 1.

3 Vgl. Ingolf Pernice, in: Horst Dreier (Hrsg.), Grundgesetz Kommentar, Tübingen 2006, Art. 23, Rn. 93.

4 Vgl. BVerfGE 123, S. 267 ff.

5 Ebenda, S. 351.

6 Ebenda.

7 Ebenda, S. $434-437$.

8 Vgl. BGBl. 2009 I, S. 3022. 
seinen in Art. 23 GG verankerten Rechten auf Unterrichtung und Stellungnahme Gebrauch macht. ${ }^{9}$ Detaillierte Bestimmungen hierzu enthielt bis zum Lissabon-Urteil die Vereinbarung zwischen Deutschem Bundestag und Bundesregierung über die Zusammenarbeit in Angelegenheiten der Europäischen Union (BBV). ${ }^{10}$ Dem Urteil zufolge sei die BBV aber weder ihrer „nicht eindeutigen Rechtsnatur noch ihrem Inhalt nach ausreichend, die verfassungsrechtlich gebotenen Beteiligungsrechte der gesetzgebenden Körperschaften am europäischen Integrationsprozess im nationalen Recht auf der Ebene des einfachen Gesetzes abzubilden und zu konkretisieren"11.

Mit der Neufassung des EUZBBG wurden daher die wesentlichen Bestimmungen der BBV in ein Gesetz überführt. Dabei konnte auf die Erfahrungen mit der BBV zurückgegriffen werden. Insbesondere machte sich der Bundestag die in zwei Monitoringberichten seiner Verwaltung ${ }^{12}$ zur Umsetzung der BBV gewonnenen Erkenntnisse zunutze, um zutage getretene Defizite zu beseitigen. ${ }^{13}$ Die Überführung der Regelungen der BBV in ein Gesetz hatte außerdem zur Folge, dass - anders als bei einer Vereinbarung - Änderungen ohne Zustimmung der Bundesregierung möglich sind.

In den ersten beiden Jahren der praktischen Anwendung erwies sich das EUZBBG als solide Grundlage für das politische Tagesgeschäft der Mitwirkung des Bundestages in EUAngelegenheiten. Es ergaben sich aber auch eine Reihe von Problemen zur Auslegung und Anwendung des Gesetzes. ${ }^{14}$ Dabei stellt sich zunächst die Frage nach der Reichweite des Begriffs „Angelegenheiten der Europäischen Union“, auf die sich die gemäß Art. 23 Abs. 2 S. 1 GG begründete und im EUZBBG konkretisierte Zusammenarbeit von Bundestag und Bundesregierung bezieht. Die Auslegung dieses Begriffs bestimmt daher sowohl die Reichweite der in $\$ \$ 3$ bis 8 EUZBBG verankerten Unterrichtungsrechte als auch das in $\$ 9$ EUZBBG geregelte Recht auf Stellungnahme. Außerdem gewinnt in jüngster Zeit auch die mögliche gerichtliche Durchsetzbarkeit des EUZBBG an Bedeutung.

9 Vgl. Wolff Heintschel von Heinegg, in: Volker Epping / Christian Hillgruber (Hrsg.), Beck'scher Online-Kommentar GG (Stand: 1. Juli 2011), Art. 23, Rn. 29; Alexander Koch, in: Andreas von Arnauld / Ulrich Hufeld (Hrsg.), Systematischer Kommentar zu den Lissabon-Begleitgesetzen, 9. Abschnitt, Rn. 1, Baden-Baden 2011.

10 Vereinbarung zwischen dem Deutschen Bundestag und der Bundesregierung über die Zusammenarbeit in Angelegenheiten der Europäischen Union (BBV) in Ausführung des $\$ 6$ des Gesetzes über die Zusammenarbeit von Bundesregierung und Deutschem Bundestag in Angelegenheiten der Europäischen Union vom 28. September 2006, in: BGBl. I, S. 2177.

11 Ebenda, S. 433.

12 Monitoringbericht vom 31. August 2007 zur Umsetzung der Unterrichtungspflichten der Bundesregierung gemäß der Vereinbarung zwischen dem Deutschen Bundestag und der Bundesregierung über die Zusammenarbeit in Angelegenheiten der Europäischen Union (BBV) sowie über die Initiative der Europäischen Kommission zur Direktzuleitung von Legislativvorschlägen und Konsultationsdokumenten (unveröffentlicht); 2. Monitoringbericht vom 9. Dezember 2008 zur Umsetzung der Unterrichtungspflichten der Bundesregierung gemäß der Vereinbarung zwischen dem Deutschen Bundestag und der Bundesregierung über die Zusammenarbeit in Angelegenheiten der Europäischen Union (BBV), Untersuchungszeitraum 1. September 2007 bis 31. August 2008 (unveröffentlicht).

13 Vgl. BT-Drs. 16/13925, S. 6.

14 Vgl. Erster Bericht über die Anwendung der Begleitgesetze zum Vertrag von Lissabon vom 17. Juni 2011 (unveröffentlicht), S. 85. 


\section{Der Gegenstand der Mitwirkungsrechte des Bundestages aus dem EUZBBG: "Angelegenheiten der Europäischen Union“}

Angelegenheiten der Europäischen Union sind jedenfalls alle Maßnahmen, die innerhalb des institutionellen Rahmens der EU vorgenommen werden. Diese bereits aus dem Wortsinn des Begriffs folgende Auslegung wurde in der praktischen Zusammenarbeit zwischen Bundestag und Bundesregierung bislang nie in Zweifel gezogen. Insbesondere erfasst sind also, wie auch der Katalog des $\$ 3$ Abs. 1 EUZBBG bestätigt, Maßnahmen der EU-Organe, auch wenn es sich nicht um Rechtssetzung handelt, wichtige Personalentscheidungen auf EU-Ebene und - wie $\$ 8$ EUZBBG klarstellt - der Bereich der Gemeinsamen Außen- und Sicherheitspolitik sowie der Gemeinsamen Sicherheits- und Verteidigungspolitik (GASP/GSVP). ${ }^{15}$

Auch Initiativen einzelner Mitgliedstaaten außerhalb des institutionellen Rahmens der EU können „Angelegenheiten der Europäischen Union“ sein, wenn sie der Verwirklichung der in den vertraglichen Grundlagen formulierten Ziele dienen. Ein aktuelles Beispiel für eine solche Initiative sind die von den Mitgliedstaaten initiierten Maßnahmen zur Stabilisierung des Euro, namentlich der EFSF ${ }^{16}$ - und der ESM-Vertrag ${ }^{17}$. Hier allein auf den zwischenstaatlichen Charakter der Zusammenarbeit abzustellen und damit die Qualifizierung als EU-Angelegenheit zu verneinen, griffe zu kurz. Die Einbeziehung der GASP/GSVP in den Begriff der Angelegenheiten der Europäischen Union verdeutlicht, dass nicht schon eine intergouvernementale Herangehensweise gegen eine solche Qualifizierung spricht. Verwiesen wird in diesem Zusammenhang auch auf das Zustandekommen von Entscheidungen im Rahmen der polizeilichen und justiziellen Zusammenarbeit in Strafsachen, die gemäß der bis zum 1. Dezember 2009 geltenden Vertragslage auf einer Zusammenarbeit der Regierungen der Mitgliedstaaten beruhte. ${ }^{18}$ Abzustellen ist vielmehr auf das Ziel einer Maßnahme. Geht es darum, ein in den Verträgen verankertes Ziel zu verwirklichen, das mit der Gemeinschaftsmethode aktuell noch nicht zu erreichen ist, somit wäre ein enger Zusammenhang mit den Verträgen bereits hergestellt. Sodann ist zu bedenken, dass Maßnahmen wie der ESM oder der EFSF nicht ergriffen werden, um eine Organisation außerhalb der

15 Vgl. Hans D. Jarass, in: ders. / Bodo Pieroth (Hrsg.), Grundgesetz Kommentar, München 2011, Art. 23, Rn. 47; Wolff Heintschel von Heinegg, a.a.O. (Fn. 9), Art. 23, Rn. 30; Ingolf Pernice, a.a.O. (Fn. 3), Art. 23, Rn. 96.

16 EFSF-Rahmenvertrag zwischen Königreich Belgien, Bundesrepublik Deutschland, Irland, Königreich Spanien, Französische Republik, Italienische Republik, Republik Zypern, Großherzogtum Luxemburg, Republik Malta, Königreich der Niederlande, Republik Österreich, Portugiesische Republik, Republik Slowenien, Slowakische Republik, Republik Finnland, Hellenische Republik und European Financial Stability Facility vom 7. Juni 2010.

17 Entwurf eines Vertrages zur Einrichtung eines Europäischen Stabilitätsmechanismus zwischen dem Königreich Belgien, der Bundesrepublik Deutschland, der Republik Estland, Irland, der Hellenischen Republik, dem Königreich Spanien, der Französischen Republik, der Italienischen Republik, der Republik Zypern, dem Großherzogtum Luxemburg, Malta, dem Königreich der Niederlande, der Republik Österreich, der Portugiesischen Republik, der Republik Slowenien, der Slowakischen Republik, der Republik Finnland (bisher unveröffentlicht); vgl. hierzu die Stellungnahme des Bundestages nach Art. 23 Abs. 3 GG Einvernehmensherstellung von Bundestag und Bundesregierung zur Ergänzung von Artikel 136 des Vertrages über die Arbeitsweise der Europäischen Union (AEUV) hinsichtlich der Einrichtung eines Europäischen Stabilitätsmechanismus (ESM); BT-Drs. 17/4880.

18 Vgl. Titel IV des Vertrags über die Europäische Union a. F. (ABl. EU Nr. C 325 vom 24. Dezember 2002, S. 1). 
EU, wie es etwa der Europarat ist, zu schaffen, sondern mit der Maßgabe einer langfristigen Integration in die bestehenden Strukturen der EU. Dies zeigt das Beispiel des so genannten Schengen-Acquis. Das Schengener Übereinkommen über den schrittweisen Abbau der Personenkontrollen an den Binnengrenzen vom 14. Juni $1985^{19}$ wurde zunächst als völkerrechtlicher Vertrag zwischen einer Gruppe von Mitgliedstaaten ${ }^{20}$ geschlossen und später in den institutionellen Rahmen der EU überführt. ${ }^{21}$ Der geltende Schengen-Acquis dient der Verwirklichung des aktuell in Art. 2 Abs. 2 EUV verankerten Ziels des Raums der Freiheit, der Sicherheit und des Rechts. Für eine Einbeziehung mitgliedstaatlicher Initiativen in den Begriff Angelegenheiten der Europäischen Union spricht schließlich, dass die parlamentarische Mitwirkung an bestimmten Maßnahmen innerhalb des institutionellen Rahmens der EU nur effektiv sein kann, wenn die Bundesregierung den Bundestag auch bei Maßnahmen außerhalb des institutionellen Rahmens der EU einbezieht. Denn werden durch die Zusammenarbeit mehrerer Mitgliedstaaten Strukturen geschaffen, die zu einem späteren Zeitpunkt in den institutionellen Rahmen überführt werden, kann der Bundestag hieran nicht mehr in vollem Umfang mitwirken. Der Begriff der Angelegenheiten der Europäischen Union kann daher auch - wie dies beim EFSF der Fall ist - Verträge der Mitgliedstaaten erfassen, an denen aus Zweckmäßigkeitserwägungen Privatrechtssubjekte beteiligt sind, denn der EFSF dient der Stabilisierung des Euro und damit einem in den Verträgen verankerten Ziel. Die privatrechtliche Rechtsform ändert hieran nichts.

Zusammenfassend sind „Angelegenheiten der Europäischen Union“ also alle Maßnahmen der Organe der EU und der Mitgliedstaaten, soweit sie der Verwirklichung der in den vertraglichen Grundlagen der EU formulierten Zielen dienen.

\section{Die Unterrichtung des Bundestages nach dem EUZBBG}

Die schriftliche und mündliche Unterrichtung der Mitglieder des Bundestages in Angelegenheiten der EU ist in $\$ \$ 3$ bis 8 EUZBBG geregelt. Sie folgt bestimmten Grundsätzen, die sich bereits aus Art. 23 Abs. 2 GG ergeben und in $\$ 4$ EUZBBG bestätigt und konkretisiert werden. Am Beginn der Unterrichtung über einen politischen Vorgang auf EU-Ebene steht regelmäßig ein Initialdokument, das in der Terminologie des $\$ 3$ EUZBBG als Vorhaben behandelt wird und dem Bundestag gemäß $\$ 6$ EUZBBG förmlich zuzuleiten ist. Informationen zu den Vorhaben erhält der Bundestag durch Übermittlung von darauf bezugnehmenden Dokumenten der Organe der EU, der anderen Mitgliedstaaten und der Bundesregierung sowie ergänzend in Form mündlicher Unterrichtung. Auch Angelegenheiten, die nicht vom Vorhabenbegriff erfasst sind, zum Beispiel Verfahren vor dem Gerichtshof der EU, sind durch das EUZBBG abgedeckt. Besondere Regeln gelten für Maßnahmen im Bereich der GASP/GSVP, die der Gesetzgeber aus dem Vorhabenbegriff herausgenommen hat. Die vertrauliche Behandlung von Dokumenten einerseits und der Zugang zu Datenbanken der EU andererseits ist in $\$ 11$ EUZBBG geregelt.

19 Vgl. GMBl. 1986, S. 79 ff.

20 Königreich Belgien, Bundesrepublik Deutschland, Französische Republik, Großherzogtum Luxemburg, Königreich der Niederlande.

21 Dazu ausführlich Holger Winkelmann, 25 Jahre Schengen: der Schengen-Acquis als integraler Bestandteil des Europarechts; Bedeutung und Auswirkung auf die Einreise- und Aufenthaltsrechte (Teil 2), in: ZAR, 30. Jg. (2010), S. 213 - 222. 


\subsection{Grundprinzipien der Unterrichtung}

Die Unterrichtung des Bundestages in Angelegenheiten der EU folgt bestimmten Prinzipien, deren Einhaltung erforderlich ist, damit das in Art. 23 Abs. 2 S. 1 GG verankerte Recht zur Mitwirkung effektiv wahrgenommen werden kann. Zwei dieser Grundprinzipien benennt Art. 23 Abs. 2 S. 2 GG: Demnach hat die Unterrichtung umfassend und zum frühestmöglichen Zeitpunkt zu erfolgen. $\$ 4$ Abs. 1 S. 1 EUZBBG ergänzt, dass fortlaufend und in der Regel schriftlich über alle Vorhaben zu informieren ist. $\$ 4$ Abs. 5 EUZBBG gibt dem Bundestag die Möglichkeit, auf die Unterrichtung zu einzelnen Vorhaben oder Gruppen von Vorhaben zu verzichten.

\section{(a) Umfassende Unterrichtung}

Eine „umfassende Unterrichtung“ verpflichtet die Bundesregierung zur Übermittlung aller ihr zugänglichen oder von ihr notfalls zu beschaffenden Informationen, die „mit der Vorbereitung, Wahrnehmung und Vollziehung von Zuständigkeiten, Befugnissen und Zielsetzungen der EU“ in einem Zusammenhang stehen. ${ }^{22}$ Dazu gehören gemäß $\$ 4$ Abs. 1 S. 2 EUZBBG die Unterrichtung über die Willensbildung der Bundesregierung, den Verlauf der Beratungen innerhalb der Organe der EU, die Stellungnahmen des Europäischen Parlaments, der Europäischen Kommission und der anderen Mitgliedstaaten sowie über die getroffenen Entscheidungen. ${ }^{23}$

Die aus dem Prinzip der umfassenden Unterrichtung folgende Pflicht zur Information über die Willensbildung der Bundesregierung steht dabei in einem Spannungsverhältnis zu dem vom BVerfG aus dem Gewaltenteilungsgrundsatz hergeleiteten „Kernbereich exekutiver Eigenverantwortung “24. Diesen hat das Gericht in seiner Rechtsprechung zu den Informationspflichten der Bundesregierung gegenüber Untersuchungsausschüssen als einen grundsätzlich nicht ausforschbaren Initiativ-, Beratungs- und Handlungsbereich definiert. Dazu gehört zum Beispiel die Willensbildung der Regierung selbst, sowohl hinsichtlich der Erörterungen im Kabinett als auch bei der Vorbereitung von Kabinetts- und Ressortentscheidungen, die sich vornehmlich in ressortübergreifenden und -internen Abstimmungsprozessen vollzieht. ${ }^{25}$

Der Schutz des Kernbereichs exekutiver Eigenverantwortung kann auch in Angelegenheiten der EU die Informationspflichten der Bundesregierung eingrenzen ${ }^{26}$, wobei aber eine wirksame parlamentarische Kontrolle möglich bleiben muss. ${ }^{27}$ Dazu reicht es nicht aus, wenn der Bundestag nur über den endgültigen Stand der Willensbildung der Bundesregierung zu einem EU-Vorhaben informiert wird. Diese Willensbildung beginnt vor Mitwirkung der Bundesregierung an einem Vorhaben, entwickelt sich im Lichte der Verhandlungen

22 Hans D. Jarass, a.a.O. (Fn. 15), Art. 23, Rn. 49; Rudolf Streinz, in: Michael Sachs (Hrsg.), Grundgesetz Kommentar, München 2009, Art. 23, Rn. 93.

23 Vgl. Ondolf Rojahn, in: Ingo von Münch / Philip Kunig (Hrsg.), Grundgesetz-Kommentar, Bd. 2, München 2001, Art. 23, Rn. 60.

24 BVerfGE 67, S. 100, S. 139.

25 Vgl. ebenda.

26 Vgl. Rupert Scholz, in: Theodor Maunz / Günter Dürig (Hrsg.), Grundgesetz. Kommentar, Bd. 3, München 2011, Art. 23, Rn. 157, S. 62; Michael Brenner, Das Gesetz über die Zusammenarbeit von Bundesregierung und Deutschem Bundestag in Angelegenheiten der Europäischen Union, in: ThürVBl., 2. Jg. (1993), S. 196, S. 200.

27 Vgl. BVerfGE 67, S. 100, S. 130; BVerfGE 124, S. 78, S. 121. 
- insbesondere in den Vorbereitungsgremien des Rates - fort und kommt vor der endgültigen Beschlussfassung im Rat, in deren Rahmen der gebildete Willen durch das Abstimmungsverhalten zum Ausdruck gebracht wird, zum Abschluss. In all diesen Stadien muss der Bundestag über den Stand der Willensbildung informiert sein, damit er seine Kontrollund Mitwirkungsrechte effektiv wahrnehmen kann. Dem Anliegen des Art. 23 Abs. 2 S. 2 GG ist also erst dann genügt, wenn die Bundesregierung den Bundestag über den jeweiligen Stand ihrer Willensbildung informiert; sie kann demgegenüber und darüber hinaus aber nicht verpflichtet werden, auch jenen gesamten internen Prozess offenzulegen, der zu dem jeweiligen Ergebnis führt oder geführt hat. ${ }^{28}$ Beruft sich die Bundesregierung auf den „Kernbereich exekutiver Eigenverantwortung “, ist sie gehalten, dies im Einzelfall zu begründen. ${ }^{29}$ Ein Recht zur Zurückbehaltung von Informationen, die bereits offiziell nach außen weitergegeben wurden, besteht allerdings in keinem Fall. ${ }^{30}$ Das bedeutet insbesondere, dass Dokumente, die einem Organ der EU auf offiziellem Wege zugeleitet wurden, auch dem Bundestag vorgelegt werden müssen.

\section{(b) Unterrichtung zum frühestmöglichen Zeitpunkt}

Eine frühestmögliche Unterrichtung erfolgt, wenn die Bundesregierung den Bundestag informiert, sobald sie, gegebenenfalls durch die Ständige Vertretung der Bundesrepublik Deutschland bei der EU, von einem Vorhaben Kenntnis erlangt. ${ }^{31}$ Die Weiterleitung von Informationen hat unverzüglich zu erfolgen, also zu einem Zeitpunkt, in dem eine Weiterleitung technisch-organisatorisch möglich ist. ${ }^{32}$ Die Pflicht zur frühestmöglichen Unterrichtung gewinnt insbesondere in Fällen, in denen Entscheidungen auf EU-Ebene in sehr kurzer Zeit getroffen werden müssen, an Bedeutung. In diesen Eilfällen ist nur durch eine umgehende Unterrichtung des Bundestages dessen Beteiligung sichergestellt.

Einige Stimmen in der Literatur wollen der Bundesregierung einen gewissen Spielraum bei der Bestimmung des Zeitpunkts der Weiterleitung von Informationen einräumen. Teilweise wird ein solcher Spielraum aus dem Kernbereich exekutiver Eigenverantwortung abgeleitet. ${ }^{33}$ Konkret bedeute dies, dass der Bundesregierung eine „angemessene Prüfungsfrist zur Klärung der Relevanz eines jeden Dokuments für den jeweiligen Adressaten zuzubilligen“34 sei. Darüber hinaus diene diese Prüfungsfrist dazu, der Bundesregierung Vorgespräche mit anderen Mitgliedstaaten und ein abgestimmtes Vorgehen zu ermöglichen. ${ }^{35}$ Der Kernbereich exekutiver Eigenverantwortung schützt allerdings nur Abstim-

28 Vgl. Hans D. Jarass, a.a.O. (Fn. 15), Art. 49, Rn. 50; Rupert Scholz, a.a.O. (Fn. 26), Rn. 157; Michael Brenner, a.a.O. (Fn. 26), S. 201.

29 Vgl. BVerfGE 67, S. 100, S. 138; BVerfGE 124, S. 78, S. 128.

30 Vgl. BVerfGE 124, S. 78, S. 140.

31 Vgl. Ingolf Pernice, a.a.O. (Fn. 3), Art. 23, Rn. 101.

32 Vgl. Clarissa Freundorfer, Die Beteiligung des Bundestages an der Sekundärrechtsetzung der Europäischen Union, Frankfurt am Main 2008, S. 65; Sandra Hansmeyer, Die Mitwirkung des Deutschen Bundestages an der europäischen Rechtsetzung, Berlin 2001, S. 213.

33 Vgl. Alexander Koch, a.a.O. (Fn. 9), 9. Abschnitt, Rn. 34; Simon Neumann, in: Andreas von Arnauld / Ulrich Hufeld (Hrsg.), a.a.O. (Fn. 9), 8. Abschnitt, Rn. 9.

34 Leila Saberzadeh, in: Andreas von Arnauld / Ulrich Hufeld (Hrsg.), a.a.O. (Fn. 9), 11. Abschnitt, Rn. 6.

35 Vgl. Simon Neumann, a.a.O. (Fn. 33), 8. Abschnitt, Rn. 9. 
mungsprozesse innerhalb der Regierung (siehe oben), von vornherein also nicht Informationen, die die Bundesregierung über Vorgänge auf EU-Ebene erlangt hat. Ein rechtliches Argument für eine Frist zur Prüfung der Relevanz eines jeden Dokuments ist daher nicht ersichtlich. Eine solche Prüfung erscheint angesichts des hohen Dokumentenaufkommens ${ }^{36}$ auch praxisfern. Soweit es um den Zeitpunkt der Unterrichtung über die Willensbildung der Bundesregierung geht, kann eine Berufung auf den Kernbereich exekutiver Eigenverantwortung nicht mehr erfolgen, sobald der interne Meinungsbildungsprozess soweit abgeschlossen ist, dass eine Position im Rat und seinen Vorbereitungsgremien vertreten werden soll (siehe oben). Würde die Bundesregierung hingegen ihre Position vor der Unterrichtung des Bundestages mit anderen Mitgliedstaaten abstimmen, könnte der Bundestag sein Recht zur Mitwirkung an der Willensbildung des Bundes nicht mehr effektiv wahrnehmen.

\section{(c) Fortlaufende Unterrichtung}

Das Prinzip der fortlaufenden Unterrichtung besagt, dass eine einmalige Information über Vorhaben nicht ausreichend ist, sondern die Bundesregierung im Falle relevanter Entwicklungen zu Aktualisierungen verpflichtet ist. ${ }^{37}$ Auf diese Weise wird dem prozesshaften Charakter der Entstehung von Gesetzgebungsakten auf EU-Ebene Rechnung getragen. Gleichzeitig wird eine Mitwirkung des Bundestages, die am aktuellen Verhandlungsstand auf EU-Ebene ausgerichtet ist, gewährleistet.

\section{(d) Schriftliche Unterrichtung}

Die Unterrichtung erfolgt gemäß $₫ 4$ Abs. 1 S. 1 EUZBBG „in der Regel schriftlich“, d.h. durch Übermittlung entsprechender Dokumente. Abweichungen vom Prinzip der Schriftlichkeit kennt das Gesetz nur als Ausnahme, zum Beispiel in $\$ 5$ Abs. 4 EUZBBG zu Sitzungen der Eurogruppe. Die mündliche Unterrichtung nach $\$ 4$ Abs. 1 S. 3 EUZBBG hat die Funktion, die schriftlichen Informationen zu ergänzen, nicht aber zu ersetzen. Sinn und Zweck der schriftlichen Unterrichtung ist, dass Informationen dem gesamten Bundestag zur Verfügung stehen. Nur dann ist gewährleistet, dass alle Abgeordneten das in Art. 38 Abs. 1 S. 2 GG gewährleistete Recht auf gleiche Teilhabe am Prozess der parlamentarischen Willensbildung ${ }^{38}$ wahrnehmen können. Bei einer mündlichen Unterrichtung, vor allem wenn diese in lediglich einem Ausschuss stattfindet, ist dies nicht gewährleistet.

\section{(e) Verzicht auf Unterrichtungen ( $\$ 4$ Abs. 5 EUZBBG)}

Gemäß $\$ 4$ Abs. 5 EUZBBG kann der Bundestag auf die Unterrichtung zu einzelnen oder Gruppen von Vorhaben verzichten, es sei denn, dass eine Fraktion oder fünf von Hundert

36 Im Zeitraum vom 27. Oktober 2009 bis zum 31. Januar 2011 erreichten den Bundestag 29.298 Dokumente auf der Grundlage des EUZBBG; vgl. Erster Bericht über die Anwendung der Begleitgesetze zum Vertrag von Lissabon vom 17. Juni 2011 (unveröffentlicht), Anlage 3.

37 Vgl. Simon Neumann, a.a.O. (Fn. 33), 8. Abschnitt, Rn. 9.

38 Vgl. BVerfGE 43, S. 142, S. 149; BVerfGE 70, S. 324, S. 354; BVerfGE 80, S. 188, S. 218; BVerfGE 96, S. 264, S. 278; BVerfGE 112, S. 118, S. 133; BVerfGE 123, S. 267, S. 342. 
der Mitglieder des Bundestages widersprechen. ${ }^{39}$ Ein entsprechender Verzicht wurde bislang nicht ausgesprochen.

\subsection{Vorhaben der Europäischen Union ( $\$ 3$ EUZBBG)}

Nach $₫ 3$ EUZBBG unterrichtet die Bundesregierung den Bundestag über alle Vorhaben, „die für die Bundesrepublik Deutschland von Interesse sein könnten“. Dieser weitgefasste und in der Praxis schwer abgrenzbare Vorhabenbegriff wurde in einem ersten Schritt durch die Anlage 1 der BBV präzisiert. Im Zuge der Neufassung des $₫ 3$ EUZBBG wurde der Vorhabenbegriff nochmals konkretisiert, erweitert und an die Erfordernisse des Vertrags von Lissabon angepasst. ${ }^{40}$ Dabei hat der Gesetzgeber dem Katalog des $₫ 3$ Abs. 1 Nr. 1 bis 14 EUZBBG das Wort „insbesondere“ vorangestellt und damit zum Ausdruck gebracht, dass die dortige Aufzählung keinen Anspruch auf Vollständigkeit erhebt. ${ }^{41}$ Eingeschränkt wird der Vorhabenbegriff aufgrund des $₫ 3$ Abs. 1 S. 2 EUZBBG, wonach Maßnahmen in den Bereichen der GASP und der GSVP in keinem Fall Vorhaben sind.

Die Identifizierung von Vorhaben erfolgt durch die Bundesregierung, die die entsprechenden Dokumente gemäß $\$ 6$ EUZBBG dem Bundestag förmlich zuleitet.

\section{(a) Der Katalog des $\$ 3$ Abs. 1 S. 1 EUZBBG}

Die in Nr. 1 und Nr. 2 aufgeführten Vorhaben betreffen Änderungen des Primärrechts der EU. Nr. 1 erfasst Vorschläge und Initiativen für Beschlüsse zur Aufnahme von Verhandlungen zu Änderungen der vertraglichen Grundlagen. Es handelt sich damit um das Initialdokument zu dem in Art. 48 EUV geregelten Verfahren. Vorschläge und Initiativen für Beschlüsse zur Aufnahme von Verhandlungen zur Vorbereitung von Beitritten zur EU sind Vorhaben im Sinne der Nr. 2. Der Ablauf des Beitrittsverfahrens ergibt sich aus Art. 49 EUV. $\$ 3$ Abs. 1 Nr. 1 und 2 sind in Zusammenhang mit $\$ 10$ EUZBBG zu sehen. Demnach hat der Bundestag vor der Aufnahme von Verhandlungen über Vertragsänderungen beziehungsweise Beitritten ein gegenüber $\$ 9$ EUZBBG gestärktes Stellungnahmerecht. Insbesondere muss die Bundesregierung Stellungnahmen des Bundestages in der Weise berücksichtigen, dass sie im Falle der Nichtdurchsetzbarkeit einer Stellungnahme sich um ein Einvernehmen nicht nur - wie im Falle des $\$ 9$ Abs. 4 EUZBBG - bemüht, sondern dieses herstellen „soll“. Damit $\$ 10$ EUZBBG seine volle Wirkung entfalten kann, ist es erforderlich, dass die Bundesregierung dem Bundestag zu einem möglichst frühen Zeitpunkt ein Dokument zuleitet, auf dessen Grundlage eine Beratung stattfinden und eine Stellungnahme abgegeben werden kann. Daher sind $\$ 3$ Abs. 1 Nr. 1 und 2 EUZBBG so zu verstehen, dass nicht abgewartet werden darf, bis ein Vorschlag für einen Beschluss zur Aufnahme von Verhandlungen zu Vertragsänderungen oder Beitritten vorliegt, sondern - sobald vorhanden - das frühestmögliche EU-Dokument, das den Beratungen über einen Beitritt oder eine Vertragsänderung zugrunde liegt, als Vorhaben behandelt und förmlich zugeleitet wird. In der Praxis könnte

39 Zur Diskussion über die Verfassungsgemäßheit dieser Bestimmung Leila Saberzadeh, a.a.O. (Fn. 34), 11. Abschnitt, Rn. 5.

40 Vgl. BT-Drs. 16/13925, S. 6.

41 Vgl. Alexander Koch, a.a.O. (Fn. 9), 9. Abschnitt, Rn. 15. 
dies zum Beispiel eine Stellungnahme der Kommission mit der Empfehlung zur Aufnahme von Beitrittsverhandlungen sein. Sollten der Bundesregierung konkrete Hinweise auf bevorstehende Beitritts- oder Vertragsänderungsverhandlungen vorliegen, ohne dass ein entsprechendes Dokument vorhanden ist, ist dem Bundestag ein Unterrichtungsdokument (zum Beispiel in Form eines Schreibens an den Bundestagspräsidenten) zu übermitteln. Diese Auslegung wird dadurch gestützt, dass in Nr. 1 und 2 nicht nur „Vorschläge“, sondern auch „Initiativen“ als Vorhabendokumente genannt sind.

Nr. 3 des Katalogs erfasst Vorschläge für Gesetzgebungsakte der EU. Der Begriff des Gesetzgebungsakts ist in Art. 289 Abs. 3 AEUV legal definiert. Danach sind solche Rechtsakte zugleich Gesetzgebungsakte, die gemäß einem Gesetzgebungsverfahren angenommen worden sind. Allen Gesetzgebungsverfahren gemeinsam ist die Beteiligung des Europäischen Parlaments, entweder in Form der Mitentscheidung oder in Form der Anhörung oder Zustimmung. ${ }^{42}$ Bei Verordnungen und Richtlinien handelt es sich daher in der Regel um Gesetzgebungsakte, während dies bei Empfehlungen und Stellungnahmen der EU-Organe nicht der Fall ist. Ob ein Beschluss in einem Gesetzgebungsverfahren zustande kommt und dementsprechend ein Gesetzgebungsakt ist, lässt sich der jeweiligen Kompetenznorm entnehmen. Beispielhaft zu nennen sind hier Beschlüsse des Rates zur Bestimmung von Aspekten des Familienrechts mit grenzüberschreitendem Bezug gemäß Art. 81 Abs. 3 Unterabsatz 2 AEUV sowie im Bereich der justiziellen Zusammenarbeit in Strafsachen nach Art. 82 Abs. 2 lit. d) AEUV. Diese Beschlüsse kommen mit Beteiligung des Europäischen Parlaments und daher in einem besonderen Gesetzgebungsverfahren zustande. Als Vorhabendokument zuzuleiten sind die vom Kommissionskollegium angenommenen Vorschläge für Gesetzgebungsakte. Sofern ein Gesetzgebungsakt auf Initiative einer Gruppe von Mitgliedstaaten, auf Empfehlung der Europäischen Zentralbank oder auf Antrag des Gerichtshofs oder der Europäischen Investitionsbank erlassen wird, ist dem Bundestag jeweils die Fassung zuzuleiten, auf deren Basis der Rat - inklusive seiner Vorbereitungsgremien - seine Beratungen aufnimmt.

Eine Neuerung gegenüber der BBV ist die Aufnahme von Verhandlungsmandaten für die Europäische Kommission zu Verhandlungen über völkerrechtliche Verträge der EU in Nr. 4. Da nach dem in Art. 218 AEUV geregelten Verfahren zur Aushandlung völkerrechtlicher Verträge der EU vor Beginn der Verhandlungen mit Drittstaaten ein Verhandlungsmandat erteilt werden muss, ist dem Bundestag nach dem EUZBBG nun die Befassung vor Aushandlung des endgültigen Vertragstexts möglich. ${ }^{43}$ Auf der Grundlage der BBV wurden dem Bundestag erst die Unterzeichnungsmandate förmlich zugeleitet. ${ }^{44}$

Praktische Probleme bereitet die Anwendung des $\$ 3$ Abs. 1 Nr. 5 EUZBBG, wonach „Beratungsgegenstände, Initiativen sowie Verhandlungsmandate und Verhandlungsrichtlinien für die Europäische Kommission im Rahmen der gemeinsamen Handelspolitik und der Welthandelsrunden" Vorhaben der EU sind. Aufgrund der sehr weiten Formulierung dieses Titels sind sehr unterschiedliche Dokumentenkategorien erfasst. So erlassen gemäß Art. 207 AEUV das Europäische Parlament und der Rat durch Verordnungen im ordentlichen Gesetzgebungsverfahren die Maßnahmen, mit denen der Rahmen für die Umsetzung der gemeinsamen Handelspolitik bestimmt wird. Die entsprechenden Dokumente sind bereits von

42 Vgl. Andreas Haratsch / Christian Koenig / Matthias Pechstein, Europarecht, Tübingen 2010, Rn. 380 .

43 Vgl. BT-Drs. 13925, S. 6.

44 Vgl. Anlage 1 zur BBV (Fn. 10). 
$\$ 3$ Abs. 1 Nr. 3 EUZBBG erfasst. Verhandlungsmandate für Abkommen mit einem Drittstaat, mehreren Drittstaaten oder internationalen Organisationen und die entsprechenden Empfehlungen dazu werden bereits von $\$ 3$ Abs. 1 Nr. 4 und 12 EUZBBG erfasst. Nicht bereits von $\$ 3$ Abs. 1 Nr. 3, 4 oder 12 EUZBBG erfasst sind Verhandlungen der Kommission gemäß Art. 207 Abs. 3 AEUV mit Drittstaaten oder internationalen Organisationen, die die Kommission im Benehmen mit einem zu ihrer Unterstützung vom Rat bestellten Sonderausschuss führt. Bei wörtlicher Auslegung des $\$ 3$ Abs. 1 Nr. 5 EUZBBG hätten dem Bundestag diese Dokumente als Beratungsgegenstände der Handelspolitik förmlich zugeleitet werden müssen, was wegen des sehr hohen Dokumentenaufkommens in der Praxis nicht geschah. ${ }^{45}$ Vielmehr entwickelte sich eine Zuleitungspraxis, auf deren Basis der Bundestag über wichtige Entwicklungen der Handelspolitik jenseits der Vorhaben aus $₫ 3$ Abs. 1 Nr. 3, 4 oder 12 EUZBBG unterrichtet wurde. Die Bundesregierung leitete dem Bundestag in diesen Fällen ausgewählte Beratungsdokumente aus dem Handelspolitikausschuss förmlich zu. Da sich daraus zumeist der Kontext der Beratungen nicht erschließt und diese nur selten in deutscher Sprache vorliegen, übermittelt die Bundesregierung zugleich Berichtsbögen gemäß $₫ 7$ Abs. 1 EUZBBG, um das jeweilige handelspolitische Vorhaben näher zu erläutern.

Dem Vorhabenbegriff unterfallen des Weiteren Mitteilungen und Stellungnahmen der Kommission (Nr. 6). Bei Stellungnahmen handelt es sich gemäß Art. 288 Abs. 5 AEUV um unverbindliche Rechtsakte. Mitteilungen sind vom Kommissionskollegium angenommene Texte, deren Inhalt rechtlich unverbindlich ist, aus denen sich aber politisch wichtige Informationen ergeben können. ${ }^{46}$

Mit Berichten der Organe der EU (Nr. 7) ist grundsätzlich die vom jeweiligen Organ angenommene Fassung gemeint. In der Unterrichtungspraxis bestanden zunächst Unsicherheiten über den in $\$ 3$ Abs. 1 Nr. 7, 8, 11 und 13 verwendeten Begriff „Organe der Europäischen Union“. Unproblematisch erfasst werden die in Art. 13 Abs. 1 AEUV genannten Organe. Fraglich ist aber, ob auch Dokumente der den Organen nachgeordneten Institutionen oder Behörden der EU in den Vorhabenbegriff einbezogen werden sollten. Beispielhaft erwähnt sei die Europäische Polizeiakademie (EPA), die im Jahr 2005 als EU-Agentur eingerichtet wurde. In der Praxis wird nunmehr auf eine generelle Unterrichtung hierzu verzichtet, um den Rahmen der förmlichen Zuleitung nicht unzweckmäßig auszuweiten. Wichtige Dokumente von Behörden und Institutionen können dennoch als Vorhaben im Sinne des $₫ 3$ Abs. 1 EUZBBG behandelt werden, weil der Katalog des $₫ 3$ Abs. 1 EUZBBG nicht abschließend ist. Dies gilt zum Beispiel für Dokumente von Eurojust ${ }^{47}$ und Europol ${ }^{48}$, bei deren Bewertung beziehungsweise Kontrolle die nationalen Parlamente gemäß Art. 85 beziehungsweise 88 AEUV zu beteiligen sind.

Bei Aktionsplänen der Organe (Nr. 8) und Politischen Programmen der Organe der EU(Nr. 11) ist zu differenzieren, welches Organ den jeweiligen Aktionsplan beziehungsweise das jeweilige politische Programm vorlegt. Bei solchen der Kommission wird das vom Kommissionskollegium angenommene Dokument als Vorhaben behandelt, da erst auf dieser Basis

45 Vgl. Erster Bericht über die Anwendung der Begleitgesetze zum Vertrag von Lissabon vom 17. Juni 2011 (unveröffentlicht), S. 14.

46 Vgl. Gunnar Pampel, Rechtsnatur und Rechtswirkungen von Mitteilungen der Kommission im europäischen Wettbewerbsrecht, in: EuZW, 16. Jg. (2005), S. 11, S. 12.

47 Vgl. BT-Drs. 17/2994, S. 3.

48 Vgl. BT-Drs. 17/2408, S. 1. 
die Beratungen in Rat und Europäischem Parlament geführt werden. Im Falle von Aktionsplänen oder politischen Programmen aus der Mitte des Rates ist dem Bundestag hingegen die Fassung als Vorhaben zuzuleiten, auf deren Basis der Rat - inklusive seiner Vorbereitungsgremien - seine Beratungen aufnimmt, damit der Bundestag seinen Einfluss auf die Verhandlungsführung der Bundesregierung im Rat geltend machen kann.

Grünbücher der Europäischen Kommission (Nr. 9) sind Diskussionsgrundlage für einen bestimmten Politikbereich; Weißbücher (Nr. 10) können, müssen aber nicht notwendigerweise an Grünbücher anknüpfen. In Weißbüchern sind die zur Diskussion stehenden Vorschläge regelmäßig konkreter als in Grünbüchern gefasst. Grün- und Weißbücher geben neben anderen Akteuren auch den nationalen Parlamenten Gelegenheit, sich zu den darin aufgeworfenen Fragen und Vorschlägen zu äußern.

Bei Empfehlungen der Europäischen Kommission (Nr. 12) handelt es sich gemäß Art. 288 Abs. 5 AEUV um unverbindliche Rechtsakte, die in der von der Kommission angenommenen Fassung als Vorhaben behandelt werden.

Im Rahmen Interinstitutioneller Vereinbarungen der Organe der EU (Nr. 13) können Rat, Parlament und Kommission gemäß Art. 295 AEUV die Einzelheiten ihrer Zusammenarbeit regeln. Diese Vereinbarungen können bindenden Charakter haben. Auch hier sind bereits die Entwürfe, auf deren Basis die Verhandlungen zwischen den Organen geführt werden, dem Bundestag zuzuleiten. Sofern ein entsprechender Entwurf vom Rat vorgelegt wird, ist bereits die Entwurfsfassung, auf deren Basis die Bundesregierung an den ratsinternen Beratungen teilnimmt, zuzuleiten.

Entscheidende Wegmarken der Haushalts- und Finanzplanung der EU (Nr. 14) sind die Verabschiedungen des mehrjährigen Finanzrahmens sowie des Jahreshaushaltsplans. In beiden Fällen ist dem Bundestag das Dokument förmlich zuzuleiten, auf dessen Basis der Rat - inklusive seiner Vorbereitungsgremien - seine Beratungen aufnimmt. Hinsichtlich des Jahreshaushaltsplans bedeutet dies, dass dem Bundestag nicht erst der Standpunkt des Rates zum Entwurf für den Haushaltsplan zugeleitet wird, sondern bereits der Vorschlag mit dem Entwurf des Haushaltsplans, den die Kommission gemäß Art. 314 Abs. 1 und 2 AEUV auf Basis der Haushaltsvorschläge der übrigen Organe aufstellt. ${ }^{49}$

\section{(b) Nicht-abschließender Charakter des Katalogs des $\$ 3$ Abs. 1 EUZBBG}

Da der in $\$ 3$ Abs. 1 Nr. 1 bis 14 aufgestellte Katalog keinen abschließenden Charakter hat, können weitere EU-Dokumente als Vorhaben behandelt werden, ohne dass es einer Änderung des EUZBBG bedarf. Der Vorhabenbegriff wird begrenzt durch $\$ 3$ Abs. 1 S. 2 EUZBBG, wonach Dokumente aus den Bereichen GASP/GSVP keine Vorhaben im Sinne des EUZBBG sind. Maßgeblich für den Inhalt des Vorhabenbegriffs ist die in Art. 23 Abs. 2 GG verankerte umfassende Unterrichtung des Bundestages ,in Angelegenheiten der Europäischen Union" 50 , weil der Vorhabenbegriff Ausgangpunkt für deren Behandlung im Bundestag ist. Daher können außerhalb der GASP/GSVP alle die EU betreffenden Maßnahmen ihrer Organe und der Mitgliedstaaten potentiell Vorhaben im Sinne des $₫ 3$ EUZBBG sein.

49 Dies erfolgt spätestens am 1. September eines jeden Jahres, das dem entsprechenden Haushaltsjahr vorausgeht, in der Praxis jedoch bereits Ende April / Anfang Mai, wenn die Kommission den Vorschlag dem Europäischen Parlament und dem Rat zuleitet.

50 Alexander Koch, a.a.O. (Fn. 9), 9. Abschnitt, Rn. 16. 


\section{(c) Maßnahmen im Bereich der GASP/GSVP}

Maßnahmen in den Bereichen der GASP und der GSVP stellen nach $₫ 3$ Abs. 1 S. 2 EUZBBG keine Vorhaben der EU im Sinne des Gesetzes dar. Für die Unterrichtung des Bundestages gilt hier $\$ 8$ EUZBBG. Es gibt jedoch bestimmte Beschlüsse des Rates, die zwar thematisch der GASP/GSVP zuzuordnen sind, aufgrund ihres institutionellen Charakters dennoch Vorhaben im Sinne des $\$ 3$ EUZBBG darstellen und daher gemäß $\$ 6$ Abs. 1 EUZBBG zuzuleiten sind. Als Beispiel sei der Vorschlag für einen Beschluss des Rates über die Organisation und die Arbeitsweise des Europäischen Auswärtigen Dienstes ${ }^{51}$ genannt.

(d) Vorhaben im Anwendungsbereich des Integrationsverantwortungsgesetzes (\$3 Abs. 2 EUZBBG)

Vorschläge und Initiativen der EU, bei denen eine Mitwirkung des Bundestages nach dem Integrationsverantwortungsgesetz erforderlich ist, sind gemäß $\$ 3$ Abs. 2 EUZBBG ebenfalls Vorhaben. Für diese gelten neben den besonderen Bestimmungen des Integrationsverantwortungsgesetzes auch die Regeln des EUZBBG zur Unterrichtung und Mitwirkung des Bundestages.

\section{(e) Förmliche Zuleitung von Vorhaben (\$ 6 Abs. 1 EUZBBG)}

Sämtliche Vorhaben leitet die Bundesregierung dem Bundestag gemäß $₫ 6$ Abs. 1 EUZBBG förmlich zu. Die Dokumente werden zusammen mit einem Anschreiben, dem erste Informationen zu entnehmen sind, übermittelt. Das Zuleitungsschreiben wird nur auf Grundlage des zuzuleitenden Dokuments erstellt, um nicht durch umfangreiche Recherchen die Zuleitung des Vorhabens an den Bundestag zu verzögern. ${ }^{52}$ Wird ein förmlich zugeleitetes Dokument bei der bundestagsinternen Überprüfung als „,beratungsrelevant“ eingestuft, wird es an die fachlich zuständigen Ausschüsse überwiesen. ${ }^{53}$

\subsection{Die Unterrichtung über Vorhaben}

Nach der förmlichen Zuleitung unterrichtet die Bundesregierung umfassend über jedes Vorhaben. Durch Übermittlung einer Vielzahl von Dokumenten der Bundesregierung und der Organe der EU sollen die Abgeordneten die aus Sicht des Bundestages wichtigsten Inhalte und Folgen der Vorhaben einschätzen können und über den jeweils aktuellen Verhandlungsstand informiert sein. Nachfolgend werden die in den verschiedenen Stadien der Entwicklung eines Vorhabens übermittelten einzelnen Dokumenttypen und die mündlichen Unterrichtungspllichten dargestellt.

51 Vgl. Beschluss des Rates vom 26. Juli 2010 über die Organisation und die Arbeitsweise des Europäischen Auswärtigen Dienstes (2010/427/EU), ABl. EU Nr. L 201 vom 3. August 2010, S. 30.

52 Vgl. BT-Drs. 16/13925, S. 8.

53 Siehe dazu ausführlich unten Abschnitt 2.7. 


\section{(a) Unterrichtung zur Folgenabschätzung}

Gemäß $\$ 4$ Abs. 2 EUZBBG umfasst die Unterrichtung der Bundesregierung auch die Abschätzung der Kommission und die der Bundesregierung vorliegenden Abschätzungen der Mitgliedstaaten zu den rechtlichen, wirtschaftlichen, finanziellen, sozialen und ökologischen Folgen eines Vorhabens. Die Folgenabschätzungen der Kommission werden nach festgelegten Leitlinien, die den Kriterien des $\$ 4$ Abs. 2 EUZBBG entsprechen ${ }^{54}$, erstellt und in der Regel im Rahmen der förmlichen Zuleitung als Addendum zu den Vorschlägen für Gesetzgebungsakte übermittelt.

\section{(b) Berichtsbogen und „Umfassende Bewertung“}

$\mathrm{Zu}$ allen Vorhaben erstellt das zuständige Fachministerium gemäß $₫ 7$ Abs. 1 EUZBBG binnen zwei Wochen nach der förmlichen Zuleitung einen Berichtsbogen. Darin gibt die Bundesregierung einen Überblick über den Inhalt des Vorhabens, eine erste Einschätzung von dessen politischen und rechtlichen Auswirkungen sowie Hinweise zum Verfahrensstand. $\mathrm{Zu}$ Vorschlägen für Gesetzgebungsakte erfolgt zudem gemäß $\$ 7$ Abs. 2 EUZBBG zwei Wochen nach Überweisung an die Ausschüsse eine so genannte Umfassende Bewertung. Zu Vorhaben im Sinne des $\$ 3$ Abs. 1 Nr. 6 bis 14 EUZBBG geschieht dies gemäß $\$ 7$ Abs. 4 EUZBBG jedoch nur auf Anforderung des Bundestages, wobei hierzu eine einzelne Fraktion, ein einzelner Abgeordneter oder die Arbeitsebene ausreicht. ${ }^{55}$ Zusätzlich zum Berichtsbogen enthält diese eine ausführliche Abschätzung der Folgen für die Bundesrepublik. Berichtsbogen und Umfassende Bewertung sollen außerdem die Abgeordneten bei der Prüfung der Vereinbarkeit des Vorhabens mit dem Subsidiaritäts- und dem Verhältnismäßigkeitsprinzip sowie bei der übrigen inhaltlichen Bewertung unterstützen.

In $\$ 7$ Abs. 3 EUZBBG ist vorgesehen, dass von der Zwei-Wochen-Frist abgewichen werden kann. Relevant ist dies bei kurzfristig auf EU-Ebene zu entscheidenden Vorhaben, wie sie insbesondere im Zuge der Finanzmarktstabilisierung des Öfteren aufgetreten sind. ${ }^{56}$ In diesem Fall verkürzt sich die Frist in dem Maße, dass eine rechtzeitige Unterrichtung des Bundestages und die Gelegenheit zur Stellungnahme nach $\$ 9$ Abs. 1 S. 1 EUZBBG gewährleistet werden kann. Nach Maßgabe des $₫ 7$ Abs. 3 S. 2 EUZBBG ist aber auch eine Verlängerung der Frist bei besonders komplexen Vorhaben möglich.

54 Vgl. Kommissiondokument SEK (2009) 92 vom 15. Januar 2009; nach diesen Leitlinien sind im Rahmen einer Folgenabschätzung folgende Fragen zu beantworten: Um welches Problem in welcher Größenordnung handelt es sich, wie entwickelt es sich weiter und wer sind die Hauptbetroffenen? Welche Meinung haben die Interessensvertreter dazu? Sollte die Union aktiv werden? Wenn ja, welche Ziele sollten zur Lösung des Problems festgelegt werden? Welche hauptsächlichen politischen Optionen bieten sich an? Welche möglichen wirtschaftlichen, sozialen und ökologischen Auswirkungen ziehen diese Optionen nach sich? Wie wirksam, effizient und kohärent tragen die jeweiligen Optionen zur Problemlösung bei? Wie können Monitoring und Evaluierung durchgeführt werden?

55 Vgl. BT-Drs. 16/13925, S. 8.

56 So wurde auf dem Treffen der Staats- und Regierungschefs des Euro-Währungsgebiets am 7. Mai 2010 vereinbart, dass „die Kommission unter Berücksichtigung der außergewöhnlichen Ereignisse einen europäischen Stabilisierungsmechanismus zur Wahrung der Finanzmarktstabilität in Europa vorschlagen [wird]. " Diesen Vorschlag nahm der Rat auf einer außerordentlichen Tagung bereits am 9. Mai 2010 an (vgl. Ratsdokumente 9602/10 und 9614/10 vom 10. Mai 2010). 


\section{(c) Dokumente von und für den Rat beziehungsweise die Kommission}

Aus $₫ 6$ Abs. 2 EUZBBG folgt, dass sämtliche als offizielle Ratsdokumente ausgefertigte Schriftstücke dem Bundestag zu übermitteln sind. Damit ist ein Großteil der in $₫ 5$ Abs. 1 Nr. 1 a) und Nr. 2 EUZBBG erfassten Dokumente bereits abgedeckt. Außerdem umfasst $\$ 6$ Abs. 2 EUZBBG die offiziellen Ratsdokumente, die nicht dem Tatbestand der $\$ 5$ Abs. 1 Nr. 1 a) und Nr. 2 EUZBBG unterfallen. Dazu gehören zum Beispiel die als Ratsdokument ausgefertigten schriftlichen Anfragen von Mitgliedern des Europäischen Parlaments.

$\$ 5$ Abs. 1 Nr. 1a) EUZBBG hat demnach insoweit eine eigene Existenzberechtigung, als auch die Dokumente des Europäischen Rates, des Rates, der informellen Ministertreffen, des Ausschusses der Ständigen Vertreter und sonstiger Ausschüsse und Arbeitsgruppen des Rates, die nicht als Ratsdokumente ausgefertigt werden, von der Bundesregierung eigeninitiativ an den Bundestag übermittelt werden.

Die gleiche Funktion erfüllt $\$ 5$ Abs. 2 EUZBBG für Berichte und Mitteilungen von Organen der EU für und über Sitzungen des Europäischen Rates, des Rates und der informellen Ministertreffen sowie des Ausschusses der Ständigen Vertreter und sonstiger Ausschüsse und Arbeitsgruppen des Rates.

$\$ 5$ Abs. 1 Nr. 1b) EUZBBG gewährleistet die Übermittlung von Dokumenten der Kommission, soweit sie an den Rat gerichtet oder der Bundesregierung auf sonstige Weise offiziell zugänglich gemacht worden sind. Dies schließt Dokumente zu delegierten Rechtsakten im Sinne des Artikels 290 AEUV ein. Gemäß Art. 290 Abs. 2 b) AEUV können delegierte Rechtsakte nur in Kraft treten, wenn das Europäische Parlament oder der Rat innerhalb der im Gesetzgebungsakt festgelegten Frist keine Einwände erhebt. Die delegierten Rechtsakte werden demnach dem Rat übermittelt, der diese als Ratsdokument ausfertigt.

Durchführungsrechtsakte im Sinne des Art. 291 AEUV sind im $₫ 5$ Abs. 1 Nr. 1b) EUZBBG hingegen nicht genannt. Grundsätzlich gelten auch hier die Unterrichtungs- und Mitwirkungsrechte des Bundestages nach Art. 23 Abs. 2 GG. In der Praxis werden dem Bundestag in einigen Fällen Durchführungsrechtsakte des Rates und der Kommission als Ratsdokumente übermittelt. Gemäß Art. 291 Abs. 3 AEUV legen das Europäische Parlament und der Rat entsprechend dem ordentlichen Gesetzgebungsverfahren durch Verordnungen im Voraus allgemeine Regeln und Grundsätze fest, nach denen die Mitgliedstaaten die Wahrnehmung der Durchführungsbefugnisse durch die Kommission kontrollieren. Die Einzelheiten hierzu regelt die am 1. März 2011 in Kraft getretene Verordnung (EU) Nr. 182/2011.

Der Gesetzgeber hat sich bei der Neufassung des EUZBBG bewusst dagegen entschieden, den Bereich der Durchführungsrechtsakte und delegierten Rechtsakte in den Vorhabenbegriff des $\$ 3$ EUZBBG aufzunehmen. Dadurch wäre der Umfang der förmlichen Zuleitung unsachgemäß ausgeweitet und überdehnt worden, ohne dass der Bundestag sein Mitwirkungsrecht angemessen würde wahrnehmen können.

(d) Berichte der Ständigen Vertretung beziehungsweise der Bundesregierung ( 55 Abs. 1 Nr. 3 EUZBBG)

Nach $\$ 5$ Abs. 1 Nr. 3 EUZBBG übersendet die Bundesregierung dem Bundestag Berichte der Ständigen Vertretung bei der EU beziehungsweise der Bundesregierung über:

- Sitzungen des Rates, der informellen Ministertreffen, des Ausschusses der Ständigen Vertreter und der Arbeitsgruppen des Rates, einschließlich der Arbeitsgruppen des Rates im Hauptstadtformat; 
- Sitzungen des Europäischen Parlaments und seiner Ausschüsse;

- die Einberufung, Verhandlungen und Ergebnisse von Trilogen ${ }^{57}$;

- Beschlüsse der Europäischen Kommission und

- geplante Vorhaben, einschließlich der Frühwarnberichte.

Die Bundesregierung ist in den Vorbereitungsgremien des Rates durch einen Mitarbeiter entweder der Ständigen Vertretung oder aus dem Fachministerium vertreten. Diesem Umstand wurde bei der Neufassung des EUZBBG Rechnung getragen, indem auch die Vorbereitungsgremien im Hauptstadtformat in die Berichtspflicht des $\$ 5$ Abs. 1 Nr. 3 EUZBBG einbezogen wurden.

Berichte über Beschlüsse der Kommission werden in der Praxis nicht gesondert verfasst. Informationen hierüber finden sich in der Regel in den Drahtberichten über die Verhandlungen in den zuständigen Ratsarbeitsgruppen. Ebenso werden keine eigenständigen Berichte über Einberufung, Verhandlungen und Ergebnisse von Trilogen übermittelt. Auch diese Informationen finden sich in der Regel in den Berichten über die Sitzungen von Ratsarbeitsgruppen. Dies ist insofern problematisch, als im Rahmen von Trilogen häufig die Weichen für eine Einigung über Legislativakte in erster Lesung gestellt werden. Wird der Bundestag darüber nicht oder nicht rechtzeitig informiert, kann er den Zeitpunkt versäumen, an dem eine Stellungnahme noch politisch sinnvoll erscheint. Diese Problematik hatte der Gesetzgeber bei der Neufassung des EUZBBG erkannt. Gleichzeitig war er sich des Problems bewusst, dass auch die Bundesregierung nicht immer über Berichte aus den Trilogen verfügt, diese also nur übersandt werden können, soweit der Ständigen Vertretung entsprechende Informationen vorliegen. ${ }^{58}$

\section{(e) Initiativen, Stellungnahmen und Erläuterungen der Bundesregierung für Organe der EU ( $\$ 5$ Abs. 2 EUZBBG)}

In $\$ 5$ Abs. 2 EUZBBG sind eine Reihe von dem Bundestag zu übermittelnden Dokumenten der Bundesregierung, der Regierungen anderer Mitgliedstaaten und des Bundesrates aufgeführt. Dazu gehören „Dokumente und Informationen über Initiativen, Stellungnahmen und Erläuterungen der Bundesregierung für Organe der Europäischen Union“. Ein Beispiel bildet die deutsch-französische Initiative zur Verschiebung des Beitritts von Bulgarien und Rumänien zum Schengen-Raum, die der Bundesminister des Innern mit seinem französischen Amtskollegen Ende Dezember 2010 in einem gemeinsamen Schreiben an die Kommission richtete. ${ }^{59}$ Eine weitere wichtige Initiative war die am 4. Februar 2011 im Rahmen der Tagung des Europäischen Rates vorgestellte deutsch-französische Initiative für einen „Pakt für Wettbewerbsfähigkeit“ (später „Euro-Plus-Pakt“). ${ }^{60}$ Sofern die Bundesregierung keine abgestimmte Entwurfsfassung, also ihre Initiative in Dokumentform übermitteln kann, muss sie dennoch zumindest Informationen darüber dem Bundestag zukommen lassen. Entscheidend ist, dass diese schriftlich und - angesichts des Erfordernisses einer umfas-

57 Gemeint sind Verhandlungen der drei am Gesetzgebungsprozess beteiligten Organe Rat, Kommission und Europäisches Parlament.

58 Vgl. BT-Drs. 16/13925, S. 7.

59 Vgl. Thomas Ludwig, Blockade gegen Rumänien und Bulgarien, in: Handelsblatt vom 22. Dezember 2010, S. 17.

60 Vgl. Schlussfolgerungen des Europäischen Rates vom 24./25. März 2011 (Ratsdokument EUCO 10/1/11, S. 5). 
senden und frühestmöglichen Unterrichtung gemäß Art. 23 Abs. 2 GG - vor der Vorstellung der Initiative bei den EU-Organen erfolgt. Würde der Bundestag erst danach informiert, könnte er nicht mehr effektiv an der Willensbildung des Bundes mitwirken.

Im Gegensatz zu Initiativen sind Stellungnahmen der Bundesregierung reaktive Dokumente, zum Beispiel schriftliche Äußerungen zu Grünbüchern, Mitteilungen oder Arbeitsprogrammen der Kommission. Eine weitere von $\$ 5$ Abs. 2 EUZBBG erfasste Dokumentenkategorie sind Sammelweisungen für den deutschen Vertreter im Ausschuss der Ständigen Vertreter. Dabei handelt es sich um interne Dokumente, die dem Bundestag vertraulich zur Verfügung gestellt werden.

Schließlich unterfallen der Vorschrift noch Initiativen der Regierungen von Mitgliedstaaten der EU. Dabei handelt es sich insbesondere um Vorschläge für Gesetzgebungsakte zur justiziellen Zusammenarbeit in Strafsachen und zur polizeilichen Zusammenarbeit auf der Grundlage von Kapitel 4 beziehungsweise 5 des Titels V AEUV, die gemäß Art. 76 AEUV auf Initiative eines Viertels der Mitgliedstaaten erlassen werden können.

Die auch in $\$ 5$ Abs. 2 EUZBBG genannten Initiativen des Bundesrates spielten in der bisherigen Praxis keine Rolle.

\section{(f) Vorbereitende Papiere und inoffizielle Dokumente (\$5 Abs. 3 EUZBBG)}

Aufgrund von $\$ 5$ Abs. 3 EUZBBG stellt die Bundesregierung dem Bundestag auf Anforderung ihr vorliegende vorbereitende Papiere der Kommission und des Rates zur Verfügung. Dies gilt auch für inoffizielle Dokumente (non papers). Für die Anforderung bedarf es keines Beschlusses des Bundestages. Es können auch einzelne Abgeordnete, deren Büros, Fraktionsangestellte sowie die Verwaltung des Bundestages entsprechende Dokumente von der Bundesregierung anfordern. ${ }^{61}$

(g) Mündliche Unterrichtung über Sitzungen bestimmter Gremien ( $\$ 5$ Abs. 4 EUZBBG)

Gemäß $\$ 5$ Abs. 4 EUZBBG unterrichtet die Bundesregierung die zuständigen Ausschüsse des Bundestages mündlich über die Sitzungen der Eurogruppe ${ }^{62}$, des Politischen und Sicherheitspolitischen Komitees ${ }^{63}$ sowie des Wirtschafts- und Finanzausschusses. ${ }^{64}$ Die Berichterstattung erfolgt in der Regel im Rahmen nichtöffentlicher Sitzungen der Ausschüsse.

$\$ 5$ Abs. 4 EUZBBG ist nur hinsichtlich der Unterrichtung über die Sitzungen der genannten Gremien selbst lex specialis zu $\$ 5$ Abs. 2 und 3 EUZBBG. Die üblichen Unterrichtungen über die Willensbildung der Bundesregierung und getroffene Entscheidungen sind vom Wortlaut des $\$ 5$ Abs. 4 EUZBBG nicht erfasst. Eine extensivere Auslegung von $\$ 5$ Abs. 4 EUZBBG würde dazu führen, dass eine Übermittlung von EU-Dokumenten, die auf Sitzungen der genannten Gremien beraten werden, gänzlich unterbleibt. Eine umfassende Unterrichtung des gesamten Bundestages, wie sie Art. 23 Abs. 2 S. 2 GG fordert, wäre dann nicht mehr gewährleistet. Dies gilt umso mehr, als dass die Eurogruppe in jüngster Zeit des Öfteren als Vorbereitungsgremium für Entscheidungen der EU-Organe diente.

61 Vgl. BT-Drs. 16/13925, S. 7.

62 Vgl. Art. 137 AEUV in Verbindung mit Protokoll Nr. 14.

63 Vgl. Art. 38 EUV.

64 Vgl. Art. 134 AEUV. 
(h) Vor- und Nachberichte zu Tagungen des Rates und des Europäischen Rates (\$5 Abs. 5 EUZBBG)

Die Bundesregierung unterrichtet nach $\$ 5$ Abs. 5 EUZBBG den Bundestag vor Tagungen des Europäischen Rates und des Rates schriftlich und mündlich zu jedem Beratungsgegenstand. Diese Unterrichtung umfasst die Grundzüge des Sach- und Verhandlungsstandes sowie die Verhandlungslinie der Bundesregierung. Nach Ratstagungen unterrichtet die Bundesregierung schriftlich und mündlich über die Ergebnisse.

(i) Unterrichtung über den Abschluss eines Gesetzgebungsverfahrens (\$ 4 Abs. 4 Nr. 2 EUZBBG)

$\$ 4$ Abs. 4 Nr. 2 EUZBBG verpflichtet die Bundesregierung, den Bundestag über den Abschluss eines Gesetzgebungsverfahrens der EU zu unterrichten. Diese Unterrichtung enthält auch eine Bewertung, ob die Bundesregierung den Gesetzgebungsakt mit den Grundsätzen der Subsidiarität und Verhältnismäßigkeit für vereinbar hält. Bei Richtlinien informiert die Bundesregierung über die zu berücksichtigenden Fristen für die innerstaatliche Umsetzung und den Umsetzungsbedarf.

Die Unterrichtung über den Abschluss eines Gesetzgebungsverfahrens und die damit einhergehende abschließende Subsidiaritätsprüfung ist unter anderem vor dem Hintergrund von Bedeutung, dass der Bundestag gemäß Art. 8 des Protokolls Nr. 2 i.V.m. Art. 263 AEUV eine Subsidiaritätsklage einleiten kann.

\subsection{Vorhabenunabhängige Unterrichtung über Angelegenheiten der Europäischen Union}

Eine Reihe von Dokumenten, die dem Bundestag nach dem EUZBBG zuzuleiten sind, lassen sich keinem einzelnen Vorhaben zuordnen. Dazu gehören insbesondere Frühwarnberichte, völkerrechtliche Verträge mit anderen Mitgliedstaaten sowie Schriftstücke im Zusammenhang mit Verfahren vor dem Gerichtshof der EU. Durch die Übermittlung dieser Dokumente werden Informationslücken geschlossen, die im Falle einer reinen vorhabenfixierten Unterrichtungspraxis entstünden.

\section{(a) Frühwarnberichte}

$\$ 4$ Abs. 3 EUZBBG bestimmt, dass die Bundesregierung den Bundestag zur Frühwarnung in der Regel schriftlich über aktuelle politische Entwicklungen der EU und geplante Vorhaben unterrichtet. Frühwarnhinweise lassen sich aus vielen Dokumenten, die nach dem EUZBBG übermittelt werden, gewinnen, zum Beispiel aus Vor- und Nachberichten zu Ratstagungen sowie Berichten über Vorbereitungsgremien. Im Kern beschränkt sich die formalisierte, schriftliche Frühwarnung der Bundesregierung jedoch auf die Übermittlung der Frühwarnberichte der Ständigen Vertretung gemäß $₫ 5$ Abs. 1 Nr. 3e) EUZBBG, die dem Bundestag dreimal jährlich übermittelt werden. Dies erscheint angesichts des in Art. 23 Abs. 2 S. 2 GG verfassungsrechtlich verankerten Prinzips der frühestmöglichen Unterrichtung nicht ausreichend. Außerdem hat der Gesetzgeber bei der Neufassung des EUZBBG die Frühwarnung 
in einem eigenen Absatz verankert, eine Unterrichtungspflicht über geplante Vorhaben eingefügt und das Erfordernis der Schriftlichkeit festgehalten. Dem lag die Erkenntnis zugrunde, dass die in der BBV verwendete Formulierung ,im Wege der politischen Frühwarnung“ der besonderen Bedeutung dieses Instruments nicht gerecht wurde. ${ }^{65}$

\section{(b) Völkerrechtliche Verträge ( $\$ 4$ Abs. 4 Nr. 1 EUZBBG)}

Gemäß $₫ 4$ Abs. 4 Nr. 1 EUZBBG unterrichtet die Bundesregierung den Bundestag über völkerrechtliche Verträge zwischen der Bundesrepublik Deutschland und Mitgliedstaaten der EU, die eine engere Kooperation in Politikbereichen normieren, die auch in die Zuständigkeit der EU fallen. Ziel dieser Verträge ist regelmäßig eine Vertiefung der Integration. Eine Regelung im Wege der Gemeinschaftsmethode scheitert jedoch entweder daran, dass noch nicht alle Mitgliedstaaten bereit für den angestrebten Integrationsschritt sind oder die Verträge keine entsprechende Rechtsgrundlage zur Verfügung stellen. Beispiele für völkerrechtliche Verträge im Anwendungsbereich des $\$ 4$ Abs. 4 Nr. 1 EUZBBG sind das Schengener Übereinkommen über den schrittweisen Abbau der Personenkontrollen an den Binnengrenzen zwischen den Vertragsparteien vom 14. Juni $1985^{66}$ und der angestrebte ESM-Vertrag. ${ }^{67}$

(c) Vertragsverletzungsverfahren/Verfahren vor dem Gerichtshof der Europäischen Union ( $\$ 4$ Abs. 4 Nr. 3 und 4 EUZBBG)

Die Bundesregierung unterrichtet sowohl im außergerichtlichen als auch im gerichtlichen Teil von Vertragsverletzungsverfahren nach Maßgabe von $\$ 4$ Abs. 4 Nr. 3 und 4 EUZBBG. Über andere Verfahren vor dem Gerichtshof der Europäischen Union wird der Bundestag gemäß $\$ 4$ Abs. 4 Nr. 4 EUZBBG informiert.

Über die beiden in Art. 258 AEUV vorgesehenen außergerichtlichen Verfahrensschritte, das Mahnschreiben und die mit Gründen versehene Stellungnahme unterrichtet die Bundesregierung durch Übermittlung der entsprechenden Schriftsätze der Kommission. Dies geschieht allerdings nur, wenn ein Vertragsverletzungsverfahren die Nichtumsetzung von Richtlinien durch den Bund betrifft. Der Begriff der Nichtumsetzung schließt aber auch die Falschumsetzung von Richtlinien ein, weil diese stets eine zumindest partielle Nichtumsetzung darstellt. Für diese Auslegung spricht auch der Sinn und Zweck der Vorschrift. Es geht darum, den Bundestag über Vertragsverletzungsverfahren, die möglicherweise gesetzgeberische Maßnahmen des Bundestages erfordern, frühzeitig zu informieren.

Vom Wortlaut des $\$ 4$ Abs. 4 Nr. 3 EUZBBG nicht erfasst sind die Antwortschreiben der Bundesregierung an die Kommission. ${ }^{68}$ Nach Angaben der Bundesregierung würden Stellungnahmen in diesem Verfahrensstadium sowohl von der Kommission als auch von den Mitgliedstaaten als vertraulich behandelt, da sich beide Seiten Spielräume zur Lösung von Konflikten im Verhandlungswege offenhalten möchten. Die geschützte Zusammenarbeit

65 Vgl. BT-Drs. 16/13925, S. 7.

66 Vgl. GMBl. 1986, a.a.O. (Fn. 19).

67 Vgl. BT-Drs. 17/4880, a.a.O. (Fn. 17).

68 Diese Auffassung hat die Bundesregierung zuletzt in einer Antwort auf eine Kleine Anfrage der Fraktion Bündnis 90/Die Grünen (BT-Drs. 17/421) bekräftigt. 
von Kommission und Mitgliedstaat sei eine unverzichtbare Voraussetzung für erfolgreiche Vermittlungsbemühungen, wie sie regelmäßig im Rahmen des Verfahrens nach Art. 258 AEUV mit dem Ziel einer Beilegung der Vorwürfe unternommen würden. Die Tatsache, dass etwa 90 Prozent der Vertragsverletzungsverfahren gegen Deutschland ohne Anrufung des Gerichtshofes beendet würden, zeige, dass die Vorgehensweise der Bundesregierung erfolgreich sei und beibehalten werden müsse. ${ }^{69}$ Allerdings ist das Argument der Vertraulichkeit seit Inkrafttreten des EUZBBG entkräftet, weil - anders als noch nach Maßgabe der BBV - der Bundestag über die Mahnschreiben Kenntnis von eingeleiteten Verfahren und den Gründen für eine mögliche Vertragsverletzung der Bundesrepublik erlangt. Trotz dieses Wissens auf Seiten des Bundestages gibt es bislang keine Hinweise, dass die Vermittlungsbemühungen im Rahmen der außergerichtlichen Verfahrensschritte des Vertragsverletzungsverfahrens weniger erfolgreich verlaufen. Angesichts des in Art. 23 Abs. 2 GG und $\$ 4$ Abs. 1 EUZBBG verankerten Grundsatzes der umfassenden Unterrichtung stellt sich daher die Frage, ob $\$ 4$ Abs. 3 Nr. 3 dahingehend angepasst werden sollte, dass nicht nur die Mahnschreiben der Kommission, sondern auch die Antwortschreiben der Bundesregierung dem Bundestag zuzuleiten sind.

Die Bundesregierung unterrichtet den Bundestag gemäß $\$ 4$ Abs. 4 Nr. 4 EUZBBG durch Übermittlung der entsprechenden Dokumente über alle im AEUV verankerten Verfahren vor dem Europäischen Gerichtshof, bei denen die Bundesrepublik Deutschland Verfahrensbeteiligte ist oder an denen sich die Bundesregierung - zum Beispiel durch Stellungnahmen - beteiligt.

\subsection{Unterrichtung über die GASP/GSVP ( $\$ 8$ EUZBBG)}

Für die Unterrichtung über die GASP und die GSVP trifft $\$ 8$ eine Sonderregelung, die die Besonderheiten dieser Politikfelder berücksichtigt. Anders als in anderen Politikfeldern werden Entwürfe für Beschlüsse im Bereich der GASP und der GSVP häufig so kurzfristig angenommen, dass für eine Beratung und Stellungnahme der nationalen Parlamente nicht ausreichend Zeit verbleibt. Aus diesem Grund übermittelt die Bundesregierung dem Bundestag gemäß $\$ 8$ Abs. 1 S. 3 eine Übersicht der absehbar zur Beratung anstehenden Rechtsakte, deren Bewertung und eine Einschätzung über den weiteren Beratungsverlauf. ${ }^{70}$ Kurz-

69 In diesem Sinne äußerte sich bereits vor Inkrafttreten des EUZBBG die Bundesregierung am 19. September 2008 auf die Schriftliche Frage des Abgeordneten Thomas Silberhorn (BT-Drs. 16/10396, S. 12).

70 Die Übersichtsliste soll nach Absprache zwischen dem Bundestag und dem Auswärtigen Amt insbesondere folgende Rechtsakte im Sinne des Art. 25 EUV im Bereich der GASP/GSVP (Aktionen, Standpunkte, Beschlüsse) umfassen: Beschlüsse des Rates über ein operatives Vorgehen der Union (vor allem GSVP-Missionen) (Art. 28, 43 EUV); Beschlüsse des Rates, in denen ein Standpunkt der Union zu einer bestimmten Frage geographischer oder thematischer Art festgelegt wird (zum Beispiel restriktive Maßnahmen) (Art. 29 EUV); Beschlüsse über die Ernennung eines EUSonderbeauftragten beziehungsweise Beschlüsse betreffend bestehende Mandate von EU-Sonderbeauftragen (zum Beispiel Verlängerung des Mandats) (Art. 33, 28 EUV); Beschlüsse im Bereich Abrüstung/Non-Proliferation (zum Beispiel Ratsbeschluss zur Unterstützung des CTBTO Verifikationssystems im Rahmen der Implementierung der EU-Strategie gegen die Proliferation von Massenvernichtungswaffen) (Art. 26, $31 \mathrm{EUV}$ ); Beschlüsse des Rates über Strategien im Rahmen der GASP (Art. 24 EUV) und Beschlüsse gemäß Art. 218 Abs. 2 AEUV (Übereinkünfte mit 
fristigen Entwicklungen trägt die Bundesregierung durch eine fortlaufende Aktualisierung der Übersicht mittels Zuleitung entsprechender Nachlieferungen an den Bundestag Rechnung. Die Unterrichtung über anstehende Maßnahmen im Rahmen der GASP/GSVP hat dabei stets vor der Beschlussfassung im Rat zu erfolgen, damit eine effektive Mitwirkung des Bundestages möglich ist.

Über Tagungen des Europäischen Rates und des Rates, die Beschlüsse und Schlussfolgerungen im Bereich der GASP/GSVP zum Gegenstand haben, unterrichtet die Bundesregierung aufgrund des $\$ 8$ Abs. 1 S. 4 EUZBBG, der auf $\$ 5$ Abs. 5 EUZBBG verweist.

Die Unterrichtung über GASP/GSVP-Dokumente von grundsätzlicher Bedeutung ist der Unterrichtung über Vorhaben angenähert. Die Dokumente sind gemäß $₫ 8$ Abs. 2 EUZBBG nach Maßgabe des $\$ 6$ Abs. 1 EUZBBG förmlich zuzuleiten, und es ist ein Berichtsbogen im Sinne des $\$ 7$ Abs. 1 EUZBBG zu erstellen. Die Anforderung bedarf keines Beschlusses des Bundestages, sondern kann von einzelnen Abgeordneten, den Fraktionen oder der Bundestagsverwaltung ausgesprochen werden. ${ }^{71}$

Gemäß $\$ 8$ Abs. 3 EUZBBG unterrichtet die Bundesregierung den Bundestag fortlaufend und zeitnah mündlich über alle relevanten Entwicklungen im Bereich der GASP/GSVP. Über Sitzungen des Politischen und Sicherheitspolitischen Komitees, das gemäß Art. 38 EUV die GASP unterstützt, unterrichtet die Bundesregierung nach $₫ 5$ Abs. 4 EUZBBG ebenfalls mündlich. Dies findet in der Regel in nichtöffentlichen Sitzungen der zuständigen Ausschüsse statt.

\subsection{Zugang zu Datenbanken, vertrauliche Behandlung von Dokumenten $(\$ 11$ EUZBBG)}

Die Bundesregierung eröffnet dem Bundestag gemäß $\$ 11$ Abs. 1 EUZBBG im Rahmen der Datenschutzvorschriften Zugang zu Dokumentendatenbanken der EU, die ihr zugänglich sind. Dem Bundestag wurde entsprechend Zugang zu ZEUS, der Datenbank des Rates, gewährt.

Gemäß $\$ 11$ Abs. 2 EUZBBG werden die EU-Dokumente grundsätzlich offen weitergegeben. Die Sicherheitseinstufung der Organe der EU über eine besondere Vertraulichkeit wird vom Bundestag beachtet. Eine für diese Dokumente oder für andere im Rahmen des EUZBBG an den Bundestag zu übermittelnde Informationen, Berichte und Mitteilungen eventuell erforderliche nationale Einstufung als vertraulich ist vor Versendung von der Bundesregierung vorzunehmen und vom Bundestag zu beachten. Die Gründe für die Einstufung sind auf Anforderung zu erläutern.

Drittländern und internationalen Organisationen) entsprechend Art. 218 Abs. 3 AEUV, soweit sich die Übereinkunft ausschließlich oder hauptsächlich auf die GASP bezieht.

71 Auf Basis bisheriger Erfahrungen verständigten sich die Fraktionen im Bundestag auf die generelle Anforderung von Dokumenten gemäß $₫ 8$ Abs. 2 S. 1 EUZBBG. Diese sind: Entwürfe von Strategien im Rahmen der GASP/GSVP; Berichte der Organe der Europäischen Union, der Hohen Vertreterin der Union für Außen- und Sicherheitspolitik zur GASP und Berichte der Strukturen der GASP von grundsätzlicher Bedeutung sowie Empfehlungen der Hohen Vertreterin für die Ermächtigung zur Aufnahme von Verhandlungen zu Übereinkommen der Union mit Drittländern oder internationalen Organisationen gem. Art. 218 Abs. 3 AEUV, sofern diese nicht bereits von $\$ 3$ Abs. 1 Nr. 4 EUZBBG erfasst sind. 
Nach $\$ 11$ Abs. 3 EUZBBG trägt der Bundestag dem besonderen Schutzbedürfnis laufender vertraulicher Verhandlungen durch eine vertrauliche Behandlung Rechnung. Neben der Beachtung der einschlägigen Bestimmungen für Verschlusssachen ergreift der Bundestag Maßnahmen zur vertraulichen Behandlung von Unterrichtungen und Dokumenten, die zwar keine Einstufung als vertraulich erfahren haben, aber dennoch nicht uneingeschränkt weitergegeben werden. So werden insbesondere die Drahtberichte der Ständigen Vertretung, die in der Regel den Stand laufender Verhandlungen wiedergeben, nur in einem passwortgeschützen Bereich des Intranets des Bundestages vorgehalten. Zudem erfolgt die Verteilung entsprechender Dokumente nur an zuständige und zugangsberechtigte Personen im Bundestag.

\subsection{Die bundestagsinterne Behandlung der Unterrichtungsdokumente}

Die Bundesregierung übermittelt dem Bundestag aufgrund ihrer Unterrichtungspflichten aus dem EUZBBG jährlich rund 25.000 Dokumente. ${ }^{72}$ Diese Menge führt im Schrifttum immer wieder zu Spekulationen über die Gefahr einer Informationsüberflutung. ${ }^{73}$ Dabei wird jedoch häufig nicht berücksichtigt, dass zum einen der Bundestag ein arbeitsteiliges Parlament ist, in dem jeder Abgeordnete bestimmte fachliche Zuständigkeiten hat und zum anderen, dass von der Vielzahl der Dokumente nur ein kleinerer Teil wichtige Informationen für das politische Handeln enthält.

Diesen Umständen trägt die bundestagsinterne Behandlung der Unionsdokumente Rechnung. Alle Vorhaben werden vor ihrer Überweisung an die fachlich zuständigen Ausschüsse auf ihre Beratungsrelevanz hin geprüft. ${ }^{74}$ Dieses Verfahren führt dazu, dass nur etwa die Hälfte der jährlich etwa 1.100 Vorhaben ihren Weg auf die Tagesordnungen der Ausschüsse findet. Bei diesem Überweisungs- und Priorisierungsverfahren sowie beim gesamten EUDokumentenmanagement werden die Fraktionen vom Europareferat der Bundestagsverwaltung unterstützt. Dort werden auch die von der Bundesregierung übermittelten Informationen für die Abgeordneten aufbereitet und an die fachlich zuständigen Ausschüsse und Arbeitsgruppen der Fraktionen weitergeleitet.

In diesem Zusammenhang ist das bundestagsinterne Folgedokumentenregime von Bedeutung. Dabei handelt es sich um Dokumente der EU-Organe, die im Beratungsprozess zu einem Vorhaben entstehen. In diese Kategorie gehören zum Beispiel Standpunkte des Europäischen Parlaments und des Rates sowie die Stellungnahmen der Kommission im Rahmen des ordentlichen Gesetzgebungsverfahrens gemäß Art. 294 AEUV. Das EUZBBG kennt den Begriff des Folgedokuments jedoch nicht. Für die praktische Anwendung der Begleitgesetze ist ein effizientes Folgedokumentenregime gleichwohl von großer Bedeutung. Gemäß $\$ 93$ a Abs. 2 S. 1 GO-BT können die Ausschüsse ,ihren Beratungen und einer Beschlussempfehlung ein Folgedokument zu dem ihnen überwiesenen Unionsdokument zugrunde legen“. Damit ist für Folgedokumente keine erneute Überweisung notwendig;

72 Vgl. Erster Bericht über die Anwendung der Begleitgesetze zum Vertrag von Lissabon vom 17. Juni 2011 (unveröffentlicht), Anlage 3.

73 Vgl. Alexander Koch, a.a.O. (Fn. 9), 9. Abschnitt, Rn. 40.

74 Vgl. $\$ 93$ Abs. 3 GO-BT. 
sie stehen den Ausschüssen sofort mit der Zuleitung an den Bundestag zur Beratung zur Verfügung. Diese Aktualität ist insbesondere für den Fall der Anpassung einer Stellungnahme gemäß $\$ 9$ Abs. 3 EUZBBG von Bedeutung.

\section{Stellungnahmen des Bundestages nach dem EUZBBG}

Das Stellungnahmerecht ist ein Instrument, um Kompetenzverschiebungen für den Bundestag auszugleichen, die durch Übertragung nationaler Zuständigkeiten, deren Ausübung der Zustimmung des Bundestages und gegebenenfalls des Bundesrates bedarf, auf die EU entstanden sind. ${ }^{75}$ Diese Kompensation erfolgt auch im Interesse des Demokratieprinzips, weil dem einzigen unmittelbar demokratisch legitimierten Verfassungsorgan des Bundes eine besondere Integrationsverantwortung zukommt, der es durch Stellungnahmen zu EU-Vorhaben gerecht werden kann. ${ }^{76}$ Eine Pflicht zur Abgabe von Stellungnahmen besteht indes nicht. ${ }^{77}$

Der Bundestag kann im Rahmen der allgemeinen Mitwirkung nach Art. 23 Abs. 2 GG zu jeder Art von Vorhaben und nicht nur zu Rechtsetzungsakten im Sinne des Art. 23 Abs. 3 GG Stellungnahmen abgeben. ${ }^{78}$ Das Verfahren dazu ist in $\$ \$ 9$ Abs. 1 bis 3 und 5 EUZBBG geregelt. Ob diese Bestimmungen auch für Stellungnahmen zur GASP und zur GSVP gelten, ist angesichts der Herausnahme dieser Bereiche aus dem Vorhabenbegriff umstritten, im Ergebnis aber wohl zu bejahen. Wenn der Bundestag eine Stellungnahme zu einem Rechtsetzungsakt nach Art. 23 Abs. 3 GG abgibt, gilt zusätzlich das in $\$ 9$ Abs. 4 EUZBBG statuierte Reglement. $\$ 10$ EUZBBG erweitert die Rechte des Bundestages im Rahmen des Stellungnahmeverfahrens im Falle einer anstehenden Aufnahme von Verhandlungen über Beitritte und Vertragsänderungen.

\subsection{Bestimmungen, die für alle Stellungnahmen gelten}

Der Wortlaut des $\$ 9$ Abs. 1 EUZBBG begrenzt den Gegenstand der Stellungnahme auf Vorhaben, zu denen nach $₫ 3$ Abs. 1 S. 2 EUZBBG Maßnahmen im Bereich der GASP und der GSVP nicht gehören. Systematische Erwägungen sprechen aber dafür, das Recht zur Stellungnahme auch auf Maßnahmen im Rahmen der GASP/GSVP zu erstrecken. Das EUZBBG regelt das Stellungnahmeverfahren nämlich im Anschluss an sämtliche Unterrichtungsrechte, also diejenigen mit Bezug zum Vorhabenbegriff in $\$ \$ 3$ bis 7 und diejenigen mit Bezug zur GASP/GSVP in $\$ 8$. Um deutlich zu machen, dass das Stellungnahmeverfahren sich nicht auf die GASP/GSVP bezieht, hätte der Gesetzgeber die Bestimmung des $₫ 8$ nach dem Stellungnahmeverfahren platzieren können. Dies spricht dafür, dass sich das Stel-

75 Vgl. Rudolf Streinz, a.a.O. (Fn. 22), Art. 23, Rn. 91; Ondolf Rojahn, a.a.O. (Fn. 23), Art. 23, Rn. 55.

76 Vgl. BVerfGE 123, S. 267, S. 351.

77 Vgl. Rupert Scholz, a.a.O. (Fn. 26), Art. 23, Rn. 161; Ingolf Pernice, a.a.O. (Fn. 3) Art. 23, Rn. 104.

78 Vgl. BT-Drs. 16/13925, S. 8. 
lungnahmerecht auf die in den $\$ \$ 3$ und 8 benannten Vorlagen bezieht. ${ }^{79}$ Für eine Einbeziehung von Maßnahmen im Bereich der GASP/GSVP als Gegenstand eines Stellungnahmeverfahrens nach dem EUZBBG spricht außerdem folgende Überlegung: $\$ 9$ Abs. 1 EUZBBG geht davon aus, dass Stellungnahmen nicht nur zu Rechtssetzungsakten nach Art. 23 Abs. 3 GG statthaft sind, sondern auch zu nichtlegislativen Maßnahmen. Ein Recht zur Stellungnahme zu nichtlegislativen Maßnahmen kann verfassungsrechtlich nur aus dem Recht zur Mitwirkung in Angelegenheiten der Europäischen Union gemäß Art. 23 Abs. 2 S. 1 GG hergeleitet werden. Erkennt man aber ein Recht zur Stellungnahme aus Art. 23 Abs. 2 S. 1 GG an, muss sich dies auf alle Angelegenheiten der EU erstrecken, also auch auf den Bereich der GASP und der GSVP. Ein so verstandenes Recht zur Stellungnahme kann einfachgesetzlich durch $\$ 9$ Abs. 1 EUZBBG nicht eingeschränkt werden. $\$ 9$ Abs. 1 EUZBBG ist also verfassungskonform dahingehend auszulegen, dass auch Maßnahmen im Bereich der GASP/GSVP in den Vorhabenbegriff des $\$ 9$ Abs. 1 S. 1 EUZBBG einbezogen werden, so dass auch hier Gelegenheit zur Stellungnahme besteht. Zu diesem Ergebnis gelangt man auch, wenn man mit guten Argumenten Art. 23 Abs. 3 GG in dem Sinne weit versteht, dass auch Maßnahmen im Rahmen der GASP und der GSVP unter den Begriff des Rechtsetzungsaktes fallen. ${ }^{80}$

Vor ihrer Mitwirkung an Vorhaben ist die Bundesregierung gemäß $₫ 9$ Abs. 1 S. 1 EUZBBG verpflichtet, dem Bundestag Gelegenheit zur Stellungnahme zu geben. Das bedeutet, dass es dem Bundestag möglich sein muss, ein Vorhaben in angemessener Zeit zu beraten, bevor er eventuell eine Stellungnahme abgibt. ${ }^{81}$ Ist die Frist seitens der EU-Organe zu kurz bemessen, muss die Bundesregierung sich dafür einsetzen, die Beratungen zu verschieben. ${ }^{82}$ Eine gesonderte Anzeige der Gelegenheit zur Stellungnahme für den Bundestag durch die Bundesregierung erfolgt in der Praxis nicht. Vielmehr besteht diese Gelegenheit in der Regel zwischen der förmlichen Zuleitung eines Vorhabens gemäß $₫ 6$ Abs. 1 EUZBBG und dem Zeitpunkt, bis zu dem eine Stellungnahme des Bundestages angemessen, also noch eine substantielle Wahrnehmung seiner Mitwirkungsrechte gewährleistet ist. Insbesondere bei eilbedürftigen Vorhaben kann dies bedeuten, dass die Bundesregierung nicht auf die Ausfertigung eines Vorhabens als Ratsdokument warten kann, sondern das frühestmöglich verfügbare vorhabeninitiierende Schriftstück zuleiten muss.

In $\$ 9$ Abs. 1 S. 2 EUZBBG ist vorgesehen, dass die Bundesregierung dem Bundestag mitteilt, bis zu welchem Zeitpunkt eine Stellungnahme angemessen erscheint. Dadurch wird es dem Bundestag ermöglicht, vor einer politischen Einigung im Rat auf die Willensbildung der Bundesregierung einzuwirken.

Eine Stellungnahme wird in Form eines einfachen Parlamentsbeschlusses vom Plenum angenommen. Das Plenum hat außerdem die Möglichkeit, den Ausschuss für die Angelegenheiten der Europäischen Union gemäß Art. 45 S. 1 und 2 GG und dem wortgleichen

79 Vgl. Birgit Daiber, Die Umsetzung des Lissabon-Urteils des Bundesverfassungsgerichts durch Bundestag und Bundesrat, in: DÖV, 63. Jg. (2010), S. 293, S. 301.

80 Vgl. Wolff Heintschel von Heinegg, a.a.O. (Fn. 9), Art. 23, Rn. 33; Frank Schorkopf, in: Rudolf Dolzer (Hrsg.), Bonner Kommentar zum Grundgesetz, Heidelberg 2011, Art. 23, Rn. 158, 153; Juliane Kokott, Kontrolle der auswärtigen Gewalt, in: DVBl., 17. Jg. (1996), S. 937, S. 942; anderer Ansicht ist Claus Dieter Classen, in: Hermann von Mangoldt / Friedrich Klein / Christian Starck (Hrsg.), Kommentar zum Grundgesetz, Bd. 2, München 2010, Art. 23, Rn. 75.

81 Vgl. Claus Dieter Classen, a.a.O. (Fn. 80), Art. 23, Rn. 80.

82 Vgl. ebenda. 
$\$ 2$ EUZBBG zu ermächtigen, im Namen des Bundestages Stellungnahmen abzugeben. Sinn dieser Regelung ist es, den Bundestag auch in sitzungsfreien Zeiten in die Lage zu versetzen, ohne Sondersitzung des Plenums eine Stellungnahme zu Vorhaben abzugeben. Dazu hat der EU-Ausschuss gemäß $₫ 93$ Abs. 3 GO-BT eine Stellungnahme der beteiligten Ausschüsse einzuholen. Will er von der Stellungnahme eines oder mehrerer Ausschüsse abweichen, soll eine gemeinsame Sitzung mit den mitberatenden Ausschüssen anberaumt werden. In eilbedürftigen Fällen können die Vorsitzenden der mitberatenden Ausschüsse entsprechend $\$ 72$ S. 2 GO-BT schriftlich abstimmen lassen. In der Praxis hat Art. 45 GG bisher kaum eine Rolle gespielt. ${ }^{83}$

Gemäß $\$ 9$ Abs. 2 S. 1 EUZBBG legt die Bundesregierung die Stellungnahmen des Bundestages ihren Verhandlungen im Rat zugrunde. Damit weicht das EUZBBG vom Wortlaut des Art. 23 Abs. 3 GG ab, wonach Stellungnahmen des Bundestages von der Bundesregierung zu „berücksichtigen“" sind. Im Schrifttum wird darauf hingewiesen, dass der Wortlaut des EUZBBG über den des Grundgesetzes hinausgeht, weshalb $\$ 9$ Abs. 2 S. 1 EUZBBG verfassungskonform als deckungsgleich mit Art. 23 Abs. 3 GG auszulegen sei. ${ }^{84}$ In der Zusammenarbeitspraxis von Bundestag und Bundesregierung haben solche Überlegungen bislang keine Rolle gespielt. Entsprechend wurde dieser Aspekt im Zuge der Verhandlungen über die Neufassung des EUZBBG nicht diskutiert und folglich auch nicht geändert. Für die Zusammenarbeit von Bundestag und Bundesregierung entscheidender dürfte es sein, dass die Bindungswirkung der Stellungnahme das Integrationsziel des Art. 23 GG und das Gebot der Zusammenarbeit nach Art. 4 Abs. 3 EUV nicht gefährden darf. Hierfür ist lediglich erforderlich, dass den Regierungsvertretern im Rat auch bei Zugrundelegung der Stellungnahme des Bundestages noch Verhandlungsspielraum bleibt. ${ }^{85}$ Der tatsächliche Einfluss einer Stellungnahme hängt ohnehin davon ab, wie konkret und mit welchem Nachdruck darin die Bundesregierung zu einem bestimmten Verhalten im Rat angehalten wird.

Die Pflicht zur fortlaufenden Unterrichtung nach $\$ 4$ Abs. 1 EUZBBG wird durch Angaben zur Berücksichtigung der Stellungnahme (\$9 Abs. 2 S. 2 EUZBBG) erweitert. Diese Angaben sind Grundlage einer etwaigen Anpassung und Ergänzung der Stellungnahme durch den Bundestag gemäß $\$ 9$ Abs. 3 S. 1 EUZBBG.

Schließlich muss die Bundesregierung den Bundestag unverzüglich schriftlich über die Beschlussfassung im Rat und die Durchsetzung seiner Stellungnahme unter Benennung von Gründen bei deren Nichtberücksichtigung (\$9 Abs. 5 S. 1 und 2 EUZBBG) unterrichten und auf Verlangen des Bundestages diese Gründe im Rahmen einer Plenardebatte erläutern ( $\$ 9$ Abs. 5 S. 3 EUZBBG). Die gesonderte Nennung in $\$ 9$ Abs. 5 S. 1 und 2 EUZBBG spricht dafür, dass entweder eine zusätzliche schriftliche Unterrichtung erfolgen muss oder aber im Falle einer Stellungnahme des Bundestages mindestens die bewährten Unterrichtungsinstrumente um diese Angaben erweitert werden müssen.

83 Vgl. Heinrich G. Ritzel / Joseph Bücker / Hermann J. Schreiner (Hrsg.), Handbuch für die Parlamentarische Praxis, Frankfurt 2009, $\$ 93 b, R n .2$.

84 Vgl. Rudolf Streinz, a.a.O. (Fn. 22), Art. 23, Rn. 101; Ondolf Rojahn, a.a.O. (Fn. 23), Art. 23, Rn. 62.

85 Vgl. Juliane Kokott, a.a.O. (Fn. 80), S. 942; Rudolf Streinz, a.a.O. (Fn. 22), Art. 23, Rn. 101. 


\subsection{Zusätzliche Regeln für Stellungnahmen zu Rechtsetzungsakten}

Bei Stellungnahmen zu Rechtsetzungsakten gemäß Art. 23 Abs. 3 S. 1 GG statuiert $\$ 9$ Abs. 4 EUZBBG zusätzlich zu den für alle Stellungnahmen geltenden Regeln weitere Pflichten der Bundesregierung. So hat sie im Rat einen Parlamentsvorbehalt einzulegen, wenn wesentliche Belange der Stellungnahme nicht durchsetzbar sind und hierüber angemessen gesondert zu berichten ( $\$ 9$ Abs. 4 S. 1 bis 3 EUZBBG). Des Weiteren bemüht sich die Bundesregierung um die Herstellung von Einvernehmen mit dem Bundestag vor der abschließenden Entscheidung im Rat (\$9 Abs. 4 S. 4 und 5 EUZBBG).

Unter Rechtsetzungsakt sind alle förmlichen Beschlüsse des Rates mit Rechtswirkung nach außen zu verstehen. ${ }^{86}$ Darunter fallen insbesondere Verordnungen und Richtlinien der EU, außerdem Beschlüsse des Rates mit Rechtswirkung nach außen; nicht von dieser Kategorie erfasst sind rechtlich unverbindliche Akte wie Empfehlungen und Stellungnahmen im Sinne des Art. 288 Abs. 5 AEUV. ${ }^{87}$ Erfasst sind ferner delegierte Rechtsakte im Sinne des Art. 290 AEUV $^{88}$ und verbindliche Maßnahmen im Bereich der GASP/GSVP. ${ }^{89}$ Damit geht der Begriff des Rechtsetzungsaktes über den Begriff des Gesetzgebungsaktes hinaus. Letzterer beschränkt sich auf Rechtsakte, an denen das Europäische Parlament beteiligt ist, während ersterer auch Beschlüsse des Rates ohne dessen Beteiligung erfassen kann.

Der gemäß $\$ 9$ Abs. 4 S. 1 EUZBBG einzulegende Parlamentsvorbehalt setzt die Nichtdurchsetzbarkeit wesentlicher Belange voraus. Welche Belange einer Stellungnahme für den Bundestag „wesentlich“ sind, ist entweder in der Stellungnahme gekennzeichnet oder im Einzelfall zu ermitteln.

Als Ort, an dem der Parlamentsvorbehalt einzulegen ist, benennt $₫ 9$ Abs. 4 S. 1 EUZBBG den Rat, nicht jedoch dessen Vorbereitungsgremien, in denen häufig wichtige Weichenstellungen auf dem Weg zu einem Gesetzgebungsakt vorgenommen werden. In den Beratungen über das EUZBBG wurde zwar über eine Pflicht der Bundesregierung zur Einlegung eines Parlamentsvorbehalts auch in den Vorbereitungsgremien des Rates diskutiert, jedoch fanden diese Überlegungen keinen Eingang in den Gesetzestext.

$\$ 9$ Abs. 4 S. 1 EUZBBG lässt zudem offen, in welchem Verhandlungsstadium die Bundesregierung einen Parlamentsvorbehalt einzulegen hat. Theoretisch ist dies bis zum formalen Abschluss der ersten Lesung möglich.

Sollten die Belange des Bundestages nach Einlegen des Parlamentsvorbehalts bis zu diesem Zeitpunkt nicht durchsetzbar sein, müsste sich die Bundesregierung gemäß $\$ 9$ Abs. 4 S. 4 EUZBBG bemühen, Einvernehmen mit dem Bundestag herzustellen, das dieser in einem gesonderten Beschluss festzustellen hat. ${ }^{90}$ Die Bindungswirkung einer Stellungnahme reicht jedoch in keinem Fall soweit, dass der Bundesregierung ein imperatives Mandat erteilt werden könnte. ${ }^{91}$ Dies folgt schon aus dem Wortlaut des Art. 23 Abs. 3 GG und wird durch $\$ 9$ Abs. 4 EUZBBG einfachgesetzlich bestätigt. Die Bundesregierung muss sich um Zu-

86 Vgl. Stefanie Schmahl, in: Helge Sodan (Hrsg.), Grundgesetz, München 2009, Art. 23, Rn. 27.

87 Vgl. Hans D. Jarass, a.a.O. (Fn. 15), Art. 23, Rn. 51.

88 Vgl. ebenda.

89 Vgl. Claus Dieter Classen, a.a.O. (Fn. 80), Art. 23, Rn. 80.

90 Vgl. Tilman Hoppe, Drum prüfe, wer sich niemals bindet - Die Vereinbarung zwischen Bundesregierung und Bundestag in Angelegenheiten der Europäischen Union, in: DVBl., 122. Jg. (2007), S. 1542.

91 Vgl. Wolff Heintschel von Heinegg, a.a.O. (Fn. 9), Art. 23, Rn. 35. 
stimmung bemühen, schuldet aber nicht den Erfolg, das Einvernehmen. ${ }^{92}$ Dadurch soll die Verhandlungsfähigkeit der Bundesregierung im Rat erhalten bleiben. ${ }^{93}$ Außerdem bleibt ihr Recht, aus wichtigen außen- und integrationspolitischen Gründen abweichende Entscheidungen zu treffen, unberührt ( $\$ 9$ Abs. 4 S. 6 EUZBBG).

Insgesamt sind die in $\$ 9$ Abs. 4 EUZBBG formulierten Bestimmungen zum Stellungnahmeverfahren nach Art. 23 Abs. 3 GG nicht optimal auf das auf EU-Ebene praktizierte ordentliche Gesetzgebungsverfahren abgestimmt. $\$ 9$ EUZBBG geht nämlich von den gemäß Art. 294 AEUV formal erforderlichen Verfahrensschritten aus, berücksichtigt aber nicht die tatsächlich im Rat stattfindenden Entscheidungsprozesse. So zeichnen sich wichtige Entscheidungen in vielen Fällen bereits in den Vorbereitungsgremien des Rates ab und werden auf der Ratstagung nur noch ohne Aussprache als so genannter „A-Punkt“ angenommen. Ein weiteres Problem ergibt sich aus dem Umstand, dass politische Weichenstellungen häufig in informellen Verfahrensschritten getroffen werden, die $\$ 9$ Abs. 4 EUZBBG nicht berücksichtigt. So werden vor der abschließenden Entscheidung im Rat (Teil-)Übereinkünfte über den Inhalt von Gesetzgebungsakten unter anderem in Form allgemeiner Ausrichtungen oder politischer Einigungen erzielt; informelle Triloge der EU-Organe werden dazu als Verhandlungsplattform genutzt. Ziel dieser informellen Prozesse ist es, die europäische Gesetzgebung so zu beschleunigen, dass bereits mit der ersten Lesung die gesetzgebenden Organe die Rechtsetzungsvorschläge in der ausgehandelten Fassung annehmen. Kommt es zur formal abschließenden Entscheidung im Rat, besteht daher in der Regel kein politischer Spielraum mehr für die Bundesregierung, sich für die Belange des Bundestages einzusetzen. Diesen Überlegungen ließe sich nur Rechnung tragen, wenn die Pflicht zur Einlegung des Parlamentsvorbehalts in $\$ 9$ Abs. 4 S. 1 EUZBBG auf den Zeitpunkt, in dem absehbar wird, dass die Position des Bundestages im Rat nicht durchsetzbar ist, vorverlagert und auch auf seine Vorbereitungsgremien erstreckt würde.

\subsection{Stellungnahmen zur Aufnahme von Beitrittsverhandlungen und Vertragsrevision}

Bezüglich der Aufnahme von Verhandlungen über den Beitritt zur EU sowie von Änderungen ihrer vertraglichen Grundlagen gelten für Stellungnahmen des Bundestages neben den Bestimmungen des $\$ 9$ EUZBBG die in $\$ 10$ Abs. 1 und 2 EUZBBG statuierten besonderen Regelungen. Dabei handelt es sich um eine gesonderte Unterrichtungspflicht gemäß $\$ 10$ Abs. 1 EUZBBG, wonach die Bundesregierung den Bundestag mit der Unterrichtung über Vorschläge und Initiativen zur Aufnahme von Beitrittsverhandlungen auf sein Recht zur Stellungnahme hinweist. Außerdem muss sich die Bundesregierung gemäß $₫ 10$ Abs. 2 S. 1 EUZBBG um ein Einvernehmen mit dem Bundestag nicht nur bemühen, sondern sie „soll“ dieses Einvernehmen herstellen.

Stellungnahmen nach $\$ 10$ EUZBBG haben auch deshalb ein höheres Gewicht, weil vor Abschluss eines Beitritts oder einer Vertragsänderung die Zustimmung des Bundestages erforderlich ist. ${ }^{94}$ Die Bundesregierung wird daher ein besonderes Interesse haben, die Stel-

92 Vgl. Tilman Hoppe, a.a.O. (Fn. 89), S. 1542.

93 Vgl. Ingolf Pernice, a.a.O. (Fn. 3), Art. 23, Rn. 104.

94 Die Zustimmung erfolgt durch ein Gesetz auf der Grundlage von Art. 23 Abs. 1 GG oder Art. 59 Abs. 1 GG. 
lungnahme des Bundestages umzusetzen, wenn sie nicht Gefahr laufen will, dass der Bundestag seine Zustimmung zu einem Beitritt oder einer Vertragsänderung verweigert.

\section{Die Durchsetzbarkeit des EUZBBG}

Der Bundestag kann sich weder in einem verwaltungs- noch in einem verfassungsgerichtlichen Verfahren direkt auf das EUZBBG berufen. Wenn der Bundestag seine Mitwirkungsrechte gegenüber der Bundesregierung geltend machen will, kann er dies nur im Rahmen eines Organstreitverfahrens tun ${ }^{95}$, in dem allerdings nur eine Verletzung des Grundgesetzes gerügt werden kann. ${ }^{96}$ Prüfungsgegenstand einer Organklage des Bundestages nach Art. 93 Abs. 1 Nr. 1 GG und $\$ 13$ Nr. 5 BVerfGG ist die Auslegung des Grundgesetzes ${ }^{97}$, nicht aber die Auslegung einfacher Gesetze. ${ }^{98}$ Das EUZBBG konkretisiert jedoch den Inhalt des Art. 23 Abs. 2 und 3 GG. ${ }^{99}$ Sieht der Bundestag seine Mitwirkungsrechte verletzt, müsste er sich also auf Art. 23 Abs. 2 und 3 GG berufen. Im Rahmen eines solches Verfahrens wäre dann zu klären, inwieweit die Rechte des Bundestages aus dem EUZBBG und dem Grundgesetz deckungsgleich sind.

\section{Ausblick: Wie könnte das EUZBBG weiterentwickelt werden?}

Grundlegend für die Reichweite des EUZBBG ist der Inhalt des Begriffs „Angelegenheiten der Europäischen Union“. Dieser umfasst neben Maßnahmen innerhalb des institutionellen Rahmens der EU auch die intergouvernementale Zusammenarbeit zwischen den Mitgliedstaaten, soweit dadurch in den Verträgen definierte Ziele der EU verfolgt werden. Da dieses Verständnis zum Teil in Zweifel gezogen wird, wäre es denkbar, eine entsprechende Definition gesetzlich zu verankern.

Das EUZBBG regelt im Einzelnen, wie die Bundesregierung über die Angelegenheiten der EU zu unterrichten hat. Soweit nach den vorangegangenen Ausführungen Unterrichtungslücken, zum Beispiel im Bereich der Vertragsverletzungsverfahren, bestehen, hat der Gesetzgeber die Möglichkeit, diese zu schließen.

Diskussionswürdig ist in diesem Zusammenhang auch das der Unterrichtungspraxis der Bundesregierung zugrunde liegende Verständnis des EUZBBG, wonach der Bundestag zwar Einblick in das Schriftgut der Bundesregierung erhält, aber in der Regel keine eigenen darüber hinausgehenden Berichte für den Bundestag gefertigt werden. Diese Frage wird insbesondere im Bereich der Frühwarnung relevant.

95 Vgl. Ondolf Rojahn, a.a.O. (Fn. 23), Art. 23, Rn. 55a.

96 Am 25. Juli 2011 reichte die Fraktion Bündnis 90/Die Grünen eine Organklage beim Bundesverfassungsgericht ein, in der eine Verletzung der Unterrichtungsrechte des Bundestages geltend gemacht wird, weil die Abgeordneten von der Regierung in einer frühen Phase der Verhandlungen über den ESM auf europäischer Ebene nicht hinreichend unterrichtet worden seien (Wolfgang Janisch, Grüne klagen wegen Euro-Rettung in Karlsruhe, in: SZ vom 25. Juli 2011, S. 4)

97 Vgl. Theodor Maunz in: ders. / Günter Dürig (Hrsg.), a.a.O. (Fn. 26), Art. 93, Rn. 15.

98 Vgl. Ralf Müller-Terpitz, in: Theodor Maunz / Bruno Schmidt-Bleibtreu / Franz Klein / Herbert Bethge (Hrsg.), Bundesverfassungsgerichtsgesetz Kommentar, $\$ 13$, Rn. 56k. 34, München 2011.

99 Eine solche Konkretisierung bereits für die BBV annehmend: Tilman Hoppe, a.a.O. (Fn. 90), S. 1541. 
Kein dringender Regelungsbedarf ist hingegen für so genannte Eilfälle ersichtlich. Das EUZBBG bietet grundsätzlich ein ausreichendes Instrumentarium, um die Beteiligungsrechte des Bundestages auch im Falle von kurzfristig auf EU-Ebene herbeizuführenden Entscheidungen zu gewährleisten. Dies kann allerdings nur gelingen, wenn die Bundesregierung den Grundsatz der frühestmöglichen Unterrichtung konsequent einhält und kurzfristig zu entscheidende Sachverhalte direkt mitteilt. In diesem Zusammenhang ist gemäß $\$ 9$ Abs. 1 S. 2 EUZBBG zu informieren, bis zu welchem Zeitpunkt eine Stellungnahme wegen der sich aus dem Verfahrensablauf ergebenden zeitlichen Vorgaben angemessen erscheint. Für die Erstellung des Berichtsbogens und der Umfassenden Bewertung gilt die verkürzte Frist des $\$ 7$ Abs. 3 EUZBBG. Ist für die Bundesregierung absehbar, dass die Beratungsplanung auf EU-Ebene - insbesondere im Rat - keine Gelegenheit zur Stellungnahme für den Bundestag zulässt, muss sie auf eine entsprechende Verschiebung der Beschlussfassung auf EU-Ebene hinwirken. Der Bundestag sollte über diese Bemühungen unterrichtet werden.

Wenig geeignet für eine Konkretisierung und Erweiterung der rechtlichen Grundlagen der Zusammenarbeit zwischen Bundestag und Bundesregierung erscheint eine Neuauflage der BBV. Gemäß $₫ 12$ EUZBBG werden „weitere Einzelheiten [...] in der Vereinbarung zwischen dem Deutschen Bundestag und der Bundesregierung geregelt". Dieser Verweis auf die BBV gibt dem Bundestag jedoch keine zusätzlichen Rechte. Soweit das EUZBBG seinem Wortlaut nach von der BBV abweicht, war damit bezweckt, die Unterrichtungsrechte des Bundestages zu erweitern oder den Anforderungen an die Rechtsförmlichkeit gerecht zu werden. Lediglich zur Ziffer VII der BBV findet sich keine Entsprechung im EUZBBG. Darin heißt es: „Die Bundesregierung unterstützt über die Ständige Vertretung und gegebenenfalls die bilaterale Botschaft im Rahmen der gegebenen Möglichkeiten und soweit erforderlich das Büro des Deutschen Bundestages in Einzelfragen im Hinblick auf seine Aufgaben." Da diese Bestimmung lediglich die Zusammenarbeit von zwei Verwaltungseinheiten beschreibt, wurde sie nicht in ein Gesetz überführt.

Abschließend sei darauf hingewiesen, dass die Wirksamkeit der im EUZBBG geregelten Mitwirkungsrechte nicht allein von der Ausgestaltung dieses Gesetzes abhängt. Eine Reihe rechtlich nicht regelbarer Faktoren spielt dafür eine Rolle. Damit ein Abgeordneter tatsächlich über alle Angelegenheiten der Europäischen Union gut unterrichtet ist, reicht es nicht aus, dass die Bundesregierung dem Bundestag die ihm gemäß EUZBBG zustehenden Dokumente zuleitet; es kommt auch auf deren Inhalt an und wie die entscheidenden Informationen im parlamentarischen Raum wahrgenommen werden. Welchen Einfluss eine Stellungnahme des Bundestages auf das Verhalten der Bundesregierung im Rat hat, hängt ebenfalls nicht allein von der gesetzlich geregelten Bindungswirkung ab. Entscheidend ist einerseits, wie konkret die darin gemachten Vorgaben sind und welche politischen Möglichkeiten die Bundesregierung hat, die Position des Bundestages im Rat durchzusetzen. Andererseits ist zu bedenken, dass auch die Bundesregierung ein vitales Interesse an einer frühzeitigen Einbeziehung des Parlaments in EU-Angelegenheiten hat. Dies liegt bei Vorhaben, die nach Art. 23 Abs. 1 GG einer Zustimmung des Bundestages und des Bundesrates bedürfen, auf der Hand. Auch bei der in vielen Fällen erforderlichen Umsetzung von EU-Recht in Bundesrecht wird sich der Bundestag bei der Verabschiedung der entsprechenden Gesetze kooperativer und wohlwollender zeigen, wenn das zugrunde liegende EU-Recht in Einklang mit seiner Position steht. 